\title{
ROUGH SET APPROACH TO SPAM FILTER LEARNING
}

\author{
A THEsIS \\ Submitted to the Faculty of Graduate Studies and Research \\ In Partial Fulfillment of the Requirements \\ FOR THE DEGREE OF \\ MASTER OF SCIENCE \\ IN \\ COMPUTER SCIENCE \\ UNIVERSITY OF REGINA
}

By

Mawuena Glymin

Regina, Saskatchewan

March 2010

(C) Copyright 2010: Mawuena Glymin 
Library and Archives

Canada

Published Heritage

Branch

395 Wellington Street

Ottawa ON K1A 0N4

Canada
Bibliotheque et

Archives Canada

Direction du

Patrimoine de l'édition

395, rue Wellington

Ottawa ON K1A ON4

Canada
Your file Votre reférence

ISBN: 978-0-494-65695-2

Our file Notre référence

ISBN: 978-0-494-65695-2
NOTICE:

The author has granted a nonexclusive license allowing Library and Archives Canada to reproduce, publish, archive, preserve, conserve, communicate to the public by telecommunication or on the Internet, loan, distribute and sell theses worldwide, for commercial or noncommercial purposes, in microform, paper, electronic and/or any other formats.

The author retains copyright ownership and moral rights in this thesis. Neither the thesis nor substantial extracts from it may be printed or otherwise reproduced without the author's permission.
AVIS:

L'auteur a accordé une licence non exclusive permettant à la Bibliothèque et Archives Canada de reproduire, publier, archiver, sauvegarder, conserver, transmettre au public par télécommunication ou par l'Internet, prêter, distribuer et vendre des thèses partout dans le monde, à des fins commerciales ou autres, sur support microforme, papier, électronique et/ou autres formats.

L'auteur conserve la propriété du droit d'auteur et des droits moraux qui protège cette thèse. $\mathrm{Ni}$ la thèse ni des extraits substantiels de celle-ci ne doivent être imprimés ou autrement reproduits sans son autorisation.
In compliance with the Canadian Privacy Act some supporting forms may have been removed from this thesis.

While these forms may be included in the document page count, their removal does not represent any loss of content from the thesis.
Conformément à la loi canadienne sur la protection de la vie privée, quelques formulaires secondaires ont été enlevés de cette thèse.

Bien que ces formulaires aient inclus dans la pagination, il n'y aura aucun contenu manquant.

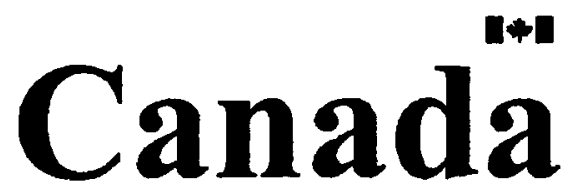




\title{
UNIVERSITY OF REGINA
}

\section{FACULTY OF GRADUATE STUDIES AND RESEARCH SUPERVISORY AND EXAMINING COMMITTEE}

\begin{abstract}
Mawuena Glymin, candidate for the degree of Master of Science in Computer Science, has presented a thesis titled, Rough Set Approach to Spam Filter Learning, in an oral examination held on December 3, 2009. The following committee members have found the thesis acceptable in form and content, and that the candidate demonstrated satisfactory knowledge of the subject material.
\end{abstract}

External Examiner: $\quad$ Dr. Luigi Benedicenti, Faculty of Engineering

Supervisor:

Dr. Wojciech Ziarko, Department of Computer Science

Committee Member:

Dr. Jing Tao Yao, Department of Computer Science

Chair of Defense:

Dr. Yang Zhao, Department of Mathematics and Statistics 


\section{Abstract}

Spammers are constantly developing problematic and more dangerous attacks designed to defeat even the most complex anti-spam filters. The rapidly evolving email spam problem is not only a real annoyance, but a significant resource drain and an important security concern.

Confronted with this issue, this thesis examines intelligent spam filter learning for email classification using machine learning techniques to learn from the content of hypertext email documents. The goal is to present a rough set approach to real time spam filtering using a probabilistic framework, the variable precision rough set model (VPRSM).

This thesis presents an elementary overview of techniques employed for spam detection via a hierarchical probabilistic decision table-based predictive data modeling. This approcah combines simple algorithms together with some heuristics to construct generalized rough approximations of spam and legitimate emails using the VPRSM. The focus here is to present a solution that can efficiently adapt to constantly evolving spam traits, and can centrally combine different technologies used to analyze emails. Research and experiments were conducted to inspect the spam problem and also explore the application of VPRSM for designing an intelligent agent for spam filtering. 


\section{Acknowledgements}

I wish to sincerely thank my thesis supervisor, Dr. W. Ziarko of the Department of Computer Science, for his encouragement and expert guidance during the entire duration of my program.

I would also like to thank my family and all others who provided support. My gratitude goes to committee members for their valuable involvement in the thesis review process. Last but not least, I thank God for giving me the will and strength to proceed until the completion of my program. 


\section{Contents}

Abstract

$\begin{array}{ll}\text { Acknowledgements } & \text { ii }\end{array}$

Table of Contents $\quad$ iii

List of Tables $\quad$ vi

List of Figures vii

$\begin{array}{lll}\text { Chapter } 1 & \text { Introduction } & 1\end{array}$

1.1 The Email Spam Problem . . . . . . . . . . . . . . . . 1

1.2 Thesis Objective ... . . . . . . . . . . . . . . 2

1.3 Thesis Organization . . . . . . . . . . . . . . . 3

$\begin{array}{lll}\text { Chapter } 2 & \text { Background } & 4\end{array}$

2.1 The Email System . . . . . . . . . . . . . . . . . . . . . . . 4

2.1.1 Addressing. . . . . . . . . . . . . . . 4

2.1 .2 Mail User Agent (MUA) . . . . . . . . . . . . 5

2.1.3 Mail Transfer Agent (MTA) . . . . . . . . . . . . . 5

2.1.4 Mail Delivery Agent (MDA) . . . . . . . . . . 5

2.1.5 Email Format . . . . . . . . . . . . . . . . . 5

2.1.6 The Flow of Email . . . . . . . . . . . . . . . . . 6

2.2 Email Spam . . . . . . . . . . . . . . . . . . . 7

2.2.1 A Brief History of Spam . . . . . . . . . . . . . . 7 
2.2.2 Origin and Categories of Spam $\ldots \ldots \ldots \ldots$

2.2.3 How Spammers Operate . . . . . . . . . . . . . . . . 13

2.2 .4 The Issues, Impact and Cost of Spam . . . . . . . . . . 16

2.2 .5 Phishing and Spam Scams _ . . . . . . . . . . . . 17

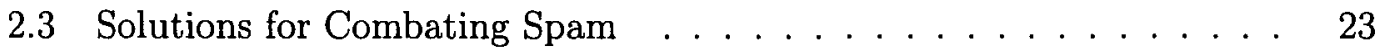

2.3.1 Legislative and Legal Action . . . . . . . . . . . . . . 23

2.3 .2 Black and White Listing . . . . . . . . . . . . . . 23

2.3 .3 Reverse DNS Lookup . . . . . . . . . . . . . . . . . . 24

2.3 .4 Content Filtering . . . . . . . . . . . . . . . . . . 24

2.3 .5 Statistical Filtering . . . . . . . . . . . . . 26

Chapter 3 Theoretical Aspects of Research 28

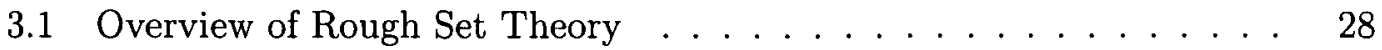

3.1 .1 Basic Concepts . . . . . . . . . . . . . . . . 29

3.1 .2 Set Approximations . . . . . . . . . . . . . . . 31

3.1.3 Rough Sets for Classification . . . . . . . . . . . . . 36

3.2 Variable Precision Rough Set Model, VPRSM _ . . . . . . . . . 39

3.2.1 Email Message Representation . . . . . . . . . . . . . . . . 39

3.2 .2 Probabilistic Knowledge . . . . . . . . . . . . . . 40

3.2 .3 Classification Knowledge . . . . . . . . . . . . . . . . . 41

3.2.4 Hierarchical Structuring of Probabilistic Decision Tables . . . 42

3.2.5 Probabilistic Decision Rules for Spam Filtering . . . . . . 47

$\begin{array}{lll}\text { Chapter } 4 & \text { Proposed Spam Detection System } & 49\end{array}$

4.1 System Design Objectives . . . . . . . . . . . . . . . 49

4.2 System Overview . . . . . . . . . . . . . . . . 50

4.3 Data Acquisition and Processing . . . . . . . . . . . . . . 52

4.3.1 Training and Testing Datasets . . . . . . . . . . 52

4.3.2 Information Extraction for Message Representation . . . . . 53

4.3.3 Attribute Analysis . . . . . . . . . . . . . . . 53

4.3.4 Reducing Attribute Domains . . . . . . . . . . . . . . . . . . . . . . . . . . . 63

4.4 HVPRS Spam Detection System . . . . . . . . . . . . . 63 
4.4.1 Data-Based Learning of Hierarchies of Probabilistic Decision Tables ....................... . . 64

4.4.2 Rule-Based Spam Classification . . . . . . . . . . . . . . . 69

4.5 System Evaluation $\ldots \ldots \ldots \ldots \ldots$

4.5 .1 Data sets Used . . . . . . . . . . . . . . 70

4.5 .2 Effectiveness Measures . . . . . . . . . . . . 71

4.5.3 Experimental Results . . . . . . . . . . . . . 72

4.5.4 Analysis of Results . . . . . . . . . . . . . 74

4.5.5 Confirmation of System Design Objectives . . . . . . . . . 78

$\begin{array}{lll}\text { Chapter } 5 & \text { Conclusion } & \mathbf{8 1}\end{array}$

5.1 Summary . . . . . . . . . . . . . . . . . 81

5.2 Future Research . . . . . . . . . . . . . . . . 82

$\begin{array}{lll}\text { Appendix A } & \text { Table of Mathematical Symbols } & 84\end{array}$

$\begin{array}{lll}\text { Appendix B } & \text { Table of Abbreviations } & 86\end{array}$

$\begin{array}{lr}\text { Bibliography } & 87\end{array}$ 


\section{List of Tables}

2.1 The ten worst spam origin countries . . . . . . . . . . . . . . 11

2.2 Exploits in spam . . . . . . . . . . . . . . 16

3.1 Example of a partial probabilistic decision table, given $l=0.1$ and

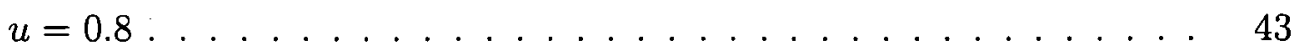

4.1 Contingency table for a binary decision category . . . . . . . . . . . 71

4.2 Sample classification experiments and reported effectiveness measures $\quad 73$

4.3 Average effectiveness measures . . . . . . . . . . . . . . . 74

A.1 Table of Symbols Used . . . . . . . . . . . . . . . . . 85

B.1 Table of Abbreviations Used . . . . . . . . . . . . . . 86 


\section{List of Figures}

2.1 The first spam. May $3,1978 \ldots \ldots \ldots \ldots \ldots$

2.2 Global spam categories, $2007 \ldots \ldots \ldots \ldots \ldots \ldots$

2.3 An example of a PayPal phishing scam. This is not an obvious spam; however investigations revealed that the seemingly reputable link displayed (https://www.paypal.com/cgi-bin/webscr?cmd= resolution-center) is different from the actual link destination. . . . . . . . . . . . . 21

2.4 An example of spam message advertising debt reduction $\ldots \ldots . .22$

3.1 Approximation of a circle using rough sets . . . . . . . . . 34

3.2 Hierarchical structuring of probabilistic decision tables $\ldots \ldots \ldots 46$

4.1 HVPRS system overview . . . . . . . . . . . . . 51

4.2 Training and classification vectors . . . . . . . . . . . 64

4.3 Timeline for training data sets . . . . . . . . . . . . 70

4.4 Timeline for testing data sets $\ldots \ldots \ldots \ldots \ldots \ldots$

4.5 Effectiveness measures for the best accurate results . . . . . . . 75

4.6 Cost analysis using the precision of legitimate emails $\ldots \ldots \ldots 75$

4.7 Effects of $(\mathrm{l}, \mathrm{u})$ on classification measures . . . . . . . . . . 77

4.8 Effects of elementary set thresholds on classification measures . . . 77

4.9 Effects of number of attributes on classification measures $\ldots \ldots 78$ 


\section{Chapter 1}

\section{Introduction}

\subsection{The Email Spam Problem}

Spamming is the abuse of electronic messaging systems to send unsolicited, undesired bulk messages. A person engaged in spamming is known as a spammer. The term spam is applicable to similar abuses in other electronic media, however this thesis deals specifically with email spam, otherwise known as unsolicited bulk (or commercial) email or junk mail.

E-mail spam [97], otherwise known as unsolicited bulk (or commercial) e-mail or junk mail may be defined as one or more nearly identical messages sent out to a large group of recipients who have not requested it and have no use for the information being relayed in the message. Because of the very low cost associated with sending emails, email spam is sent to millions of recipients daily over the Internet.

Today email spam is sent in large volumes by means of dynamic zombie botnets (networks of compromised computers remotely controlled and suitably configured to suit the desires of spammers and hackers). These concerns have indeed created some issues for many email users, including increased cost for network bandwidth and storage, identity theft, increase in viruses and security vulnerabilities [41]. Consequently, new approaches are preferred to deal with the email spam issue.

There are a number of existing solutions for dealing with spam, however current prevention techniques are hardly keeping up with spams increasing complexity and rapidly evolving threats. For example, there are legislative and legal actions, black/white listing, content based filtering and statistical filtering, amongst others 
$[98,75]$. Generations of spam filters have emerged over the years to deal with the spam issue similarly to how human intelligence would analyze email content to determine if it is spam or not. Most of these filters succeed to some point in discriminating between spam and legitimate emails, however they require manual intervention. For example, simple content based methods require human efforts to build lists of characteristics and their scores. Over the last five years, statistical filters have gained more attention as they are able to tweak themselves, improving efficiency over time with less manual intervention. Even such filters have their limitations as spammers still manage to evade them by using various exploiting techniques [32]. Consequently, novel approaches are desired to deal with the ever-increasing flood of spam and the persistent attempts by spammers to break the existing anti-spam barriers.

Many of these solutions have encouraged the methodology presented in this paper, with an emphasis on supervised machine learning algorithms in the context of statistical spam filtering.

\subsection{Thesis Objective}

In this thesis, I will report on research and related experiments conducted to examine the application of rough set/variable precision rough set model for adaptive rule-based spam filtering. The main idea of this approach is to use a hierarchical-based variable precision rough set (HVPRS) learning algorithm to analyze email objects to recognize or learn patterns representing statistical trends in data. HVPRS employs the variable precision rough set model to discover interesting patterns via the construction of a hierarchy of probabilistic decision tables $[57,116,105,103]$. Identified patterns are interpreted into rules which are used to filter email for categorical classification

This approach has the potential to lead to a more powerful application technology for a better solution to the email spam problem. It broadens content-based spam filtering, with a system that has a real advantage to aggregate a number of investigative techniques to determine if an email is spam or legitimate. It also offers the ability to control the progress of learning and allows system administrators the ease 
of incorporating the latest data from new evolving spam. It takes the whole message into account for rule derivation, and is able to detect small variance in the inherent properties existing between different email objects.

\subsection{Thesis Organization}

The rest of this thesis is organized into four chapters:

- Chapter 2: Background. This chapter presents the background overview for this thesis; discussing the email system, spam and solutions to deal with the high volumes of spam email messages. The reader's knowledge regarding the background information discussed is not assumed.

- Chapter 3. Theoretical Aspects of Research. This chapter focuses on the fundamental aspects of the proposed spam detection system, by presenting an overview of the original rough set theory as proposed by Pawlak [55]. The chapter then explains Ziarko's probabilistic extension of the original theory, known as the variable precision rough set model [116].

- Chapter 4. Proposed Spam Detection System. Based on chapters 2 and 3, a detailed description of the proposed spam detection system is presented. This includes system design objectives, components, implementation, evaluation and verification.

- Chapter 5. Conclusion. This chapter concludes the thesis by providing a summary of the experimental research and then outlines future research and system enhancements. 


\section{Chapter 2}

\section{Background}

This chapter provides the reader with the background information regarding the email system, spam email, as well as solutions to deal with the spam problem.

Section 2.1 discusses the internet email system with regards to the format and flow of email using internet mailing agents. The definition of email spam is discussed in section 2.2 , along with various details of spam including history, origin and categories, spammers operations, issues, impact, cost and types of scams. Lastly, section 2.3 addresses some existing solutions available for dealing with email spam.

\subsection{The Email System}

Electronic mail or email is a system of collaborative agents that provide the technology to compose, transmit, store and retrieve electronic messages. This service is based on the Simple Mail Transfer Protocol (SMTP) [39] or Internet Protocols (IP) [39] for intranet systems. The subsections following describe the format and flow of email, using various internet mailing agents also addressed below.

\subsubsection{Addressing}

An email address is a string of the form user@domain.com that specifies the location to which email messages should be delivered. The local-part of the address is that before the @ sign and is often the recipient's username; the part after the @ sign is usually referred to as the host or domain name identifying a server on the internet. The Internet Engineering Task Force [76], RFC (request for comments) 2822 
[9], defines the format of an email address, permitting it to consist of only a subset of the ASCII characters.

\subsubsection{Mail User Agent (MUA)}

A Mail User Agent (MUA) is a program that allows a user to read, compose and send email messages. This program is often referred to as an email client; it serves as a front-end interface for accessing messages either downloaded onto a local machine or residing on a remote server. Examples include Microsoft Outlook Express, Mozilla Thunderbird, and some web-based clients like Hotmail, Yahoo and Gmail.

\subsubsection{Mail Transfer Agent (MTA)}

A Mail Transfer Agent (MTA) is a software agent used to transfer email messages between relay computers from source to destination. In the context of the Domain Name System (DNS) [1], it is also known as a mail transport agent, mail server, or a mail exchange.

\subsubsection{Mail Delivery Agent (MDA)}

A Mail Delivery Agent (MDA) is program that receives incoming electronic messages and delivers them to destination mailboxes. Procmail is an example of a Unixbased MDA.

\subsubsection{Email Format}

RFC 822 [9] and RFC 2882 [9] specify the standard for email messages. As discussed in RFC 2821 [9], SMTP transports a mail object which contains an SMTP envelope and content. The envelope consists of source and destination email addresses amongst other protocol options. The content has two parts namely header and body:

1. The header represents a collection of field/value pairs that provide summary information about the sender, receiver, and other details of the email. Message headers consist of the following mandatory fields - from, to, subject and date. 
The from field specifies the composer of the message. Reply-to is used to optionally specify the address where any replies should be sent. To, carbon copy (Cc) and blind carbon copy (Bcc) specify message recipients. Subject contains a brief summary about message contents. The date field specifies the date and time the composer sent the message. All field names start in the first character of a line, followed by a ":", then by the value of that field. All field names and their values are restricted to 7-bit ASCII characters [9]. Non-ASCII values may be represented using Multipurpose Internet Mail Extensions (MIME) encoded words [35].

2. The body represents the main part of an email message containing the actual message body. If structured, then bodies are represented according to MIME [35].

\subsubsection{The Flow of Email}

This section describes the delivery of email using the hardware infrastructure of Internet Service Providers (ISPs) and/or Email Service Providers (ESPs) to facilitate routing and relay services. Given Michelle as the sender of an email message, and Colette as the recipient, the following describes the flow from source to destination:

1. Michelle (michelle@hostA.com) composes an email message, specifying Colette as the recipient by providing her email address colette@hostB.com, as a destination address.

2. After composing the message Michelle will select the send option to deliver it. Upon sending, her MUA (e.g. Outlook Express) will format the message and transport it to her domain's local MTA (e.g. smtp.hostA.com), using email standards and SMTP [39] respectively.

3. Given the domain name (hostB.com) of the destination email address as provided by SMTP, the MTA (smtp.hostA.com) performs a DNS look up on this domain to find the mail exchange server, (e.g. mxs.hostB.com), accepting the message on behalf of the recipient's domain. 
4. stmp.hostA.com forwards the message to mxs.hostB.com using SMTP. A MDA, (software residing on mxs.hostB.com) will accept Colette's incoming email and will either deliver it to her mailbox if she has her account on a local machine; or will forward it to another SMTP server if her account is on a remote server.

5. Colette uses her email client to access her message from Michelle. Two popular standard protocols used to retrieve email from a remote server over a TCP/IP connection are the Post Office Protocol (POP3) [35] and Internet Message Access Protocol (IMAP) [43]. She may also access her mails by logging directly into a remote mail server (mxs.hostB.com), via a web-based interface.

\subsection{Email Spam}

Formulating a precise definition for spam is difficult, but for an understanding of the problem I define email spam, otherwise known as unsolicited bulk (or commercial) email or junk mail as: one or more nearly identical messages, possibly fraudulent or malicious, that is sent out to a large group of recipients who have not requested it and most likely have no real use for the information being relayed in the message. Because of the very low cost associated with sending emails, spammers are able to send millions of emails daily.

\subsubsection{A Brief History of Spam}

The dependent growth of email around the world has encouraged the steady escalation of spam, one of the biggest problems facing the internet today. In attempts to provide readers a conceptual clarity on the phases of the spam problem I will describe it using the phases as outlined in an Information \& Communications Technology Law Journal article [29]. Steve Hedley, author of this article describes spam to have evolved from being a simple problem of internet etiquette, into a more serious problem of individual rights and crime, calling for technological and legislative combating solutions. Today, spam is transmitted using sophisticated techniques to get around spam filters. Below are the phases of the spam problem. 


\section{Spam as a problem of netiquette}

The earliest phase of spam occured when the internet was lawless, as law enforcement authorities and courts were relatively ignorant of the internet.

The first incident of spam email was dispatched to about 320 mail boxes connected to the ARPANET on 3 May 1978. Gary Thuerk was responsible for this attempt to spam every email account on the West Coast of the United States [97]. The message body of the first spam email is shown in figure 2.1. Later, on 12 April 1994, lawyers from Arizona, Laurence Canter and Martha Siegel advertised their immigration advise service by posting the first massive commercial Usenet spam. The "Green Card" spam (relating to a US government Green Card Lottery program) was however the first commercial Usenet spam $[97]$. 


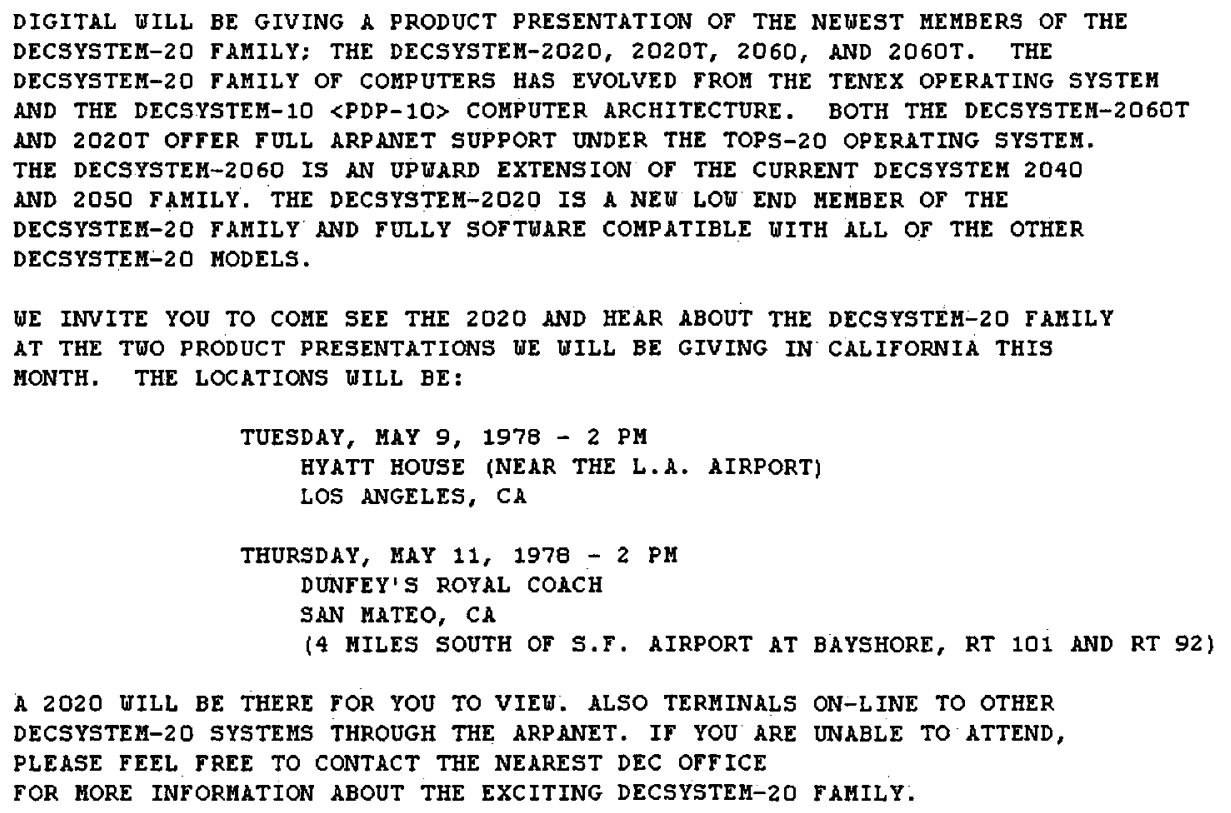

Figure 2.1: The first spam. May 3, 1978

\section{Spam as a problem of individual rights}

Hedley states that as spam continued to grow, recipients of spam complained and looked to their ISPs for a solution to the problem. Thus spam was perceived as a distinct problem to ISPs who began the initial attempts to find technical solutions to spam. Solutions included filtering software, blacklisting of spam sources, and creating umbrella organizations to deal with the problem. At this point legal action was still out of the question. Spam was insignificantly used by criminals and was arguably nothing but a nuisance, although it was a huge expense for ISPs. Nonetheless, ISPs were not prevented from taking civil action against spammers given a plausible theory of liability. Some success was attained by treating spam as an infringement of individual rights.

\section{Spam as a problem of organized crime}

At this phase of the problem, there was an increase in the diversification and volumes of spam thus leading to fraudulent and malware messages. ISPs responded by increasing their technical efforts to identify and delete spam before 
reaching their customers. On the legal side, the escalating cries for anti-spam laws resulted in regulations to be considered. The CAN-SPAM ACT [75] came into force on January 1, 2004 - and finally spammers could be successfully prosecuted. Soon after the enactment of CAN-SPAM, Jeremy Jaynes was convicted in November 2004 for sending bulk, unsolicited email with forged headers. He was convicted and sentenced to nine years in jail for using servers located in Virginia $[27,80]$.

\section{The rise of the botnet}

Previously spammers used a handful of IP addresses making it relatively easy to trace and block their activities; however the majority of spam on the internet today is distributed by botnets [61]. A botnet is a network of many broadband zombie computers that have been commandeered by hackers for use in spam runs, distributed denial-of-service attacks or malware installation. These zombie computers have been silently broken into and infected with malware giving an unauthorized remote user the ability to control them. Zombies are controlled by an Internet Relay Chat (IRC) server installed illegally on a public IRC network [34].

Today, spammers do not need to send out spam messages from one machine instead they distribute their attack using multiple computers. Spammers can render themselves untraceable as each zombie sends out just a few messages, rather than having a large volume of emails coming from a single IP. The rise of botnets has driven spam to be a vital business concern for many companies wishing to keep email as a safe and useful communications tool.

Readers should note that as today's spamming techniques rapidly evolve into mechanisms for transmitting a plethora of spam over the internet, so does legal and technical attempts to fight the problem.

\subsubsection{Origin and Categories of Spam}

- Origin 


\begin{tabular}{|l|l|}
\hline Rank & Country \\
\hline 1 & United States \\
2 & China \\
3 & Russia \\
4 & United Kingdom \\
5 & Japan \\
6 & South Korea \\
7 & Germany \\
8 & Netherlands \\
9 & Canada \\
10 & Taiwan \\
\hline
\end{tabular}

Table 2.1: The ten worst spam origin countries

The origin of an email refers to the geographical location of the original computer to send the message. This location does not consider the sender's country of residence, nor the country which hosts the products being advertised. Table 2.1 shows the Spamhaus' ranking of the world's worst spam haven countries as of April 2007 [72].

\section{- Categories of Spam}

In a recent spam report generated by Symantec Messaging and Web Security [77], they presented spam category data collected from classifications of messages passing through the Symantec Probe Network. Figure 2.2 shows these spam categories as of January 2007 and their relative occurrence. These categories are:

1. Adult: Contains material suitable only for persons of legal age. E.g. pornography, dating services, etc.

2. Fraud: Phishing messages attempting to trick users into revealing personal information. E.g. account notification, credit card verification, etc.

3. Health: Health related products and services. E.g. dietary supplements, disease prevention, etc. 
4. Internet: Internet/computer-related. E.g. web hosting, domain name registration, email marketing, etc.

5. Leisure: Containing prize offers and discounted leisure activities. E.g. vacation deals, online casinos, etc.

6. Products: General products and services. E.g. manufactured goods, services, etc.

7. Scams: Contains fraudulent and intentionally misguiding information. E.g. Investment scams, pyramid schemes, etc.

8. Financial: Relating to money. E.g. credit cards, refinancing, etc.

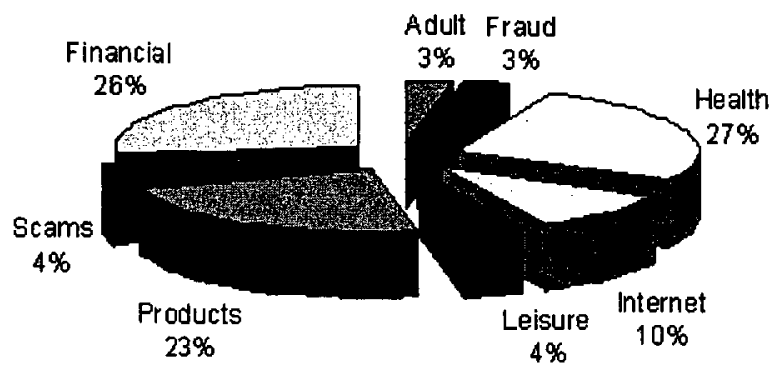

Figure 2.2: Global spam categories, 2007 


\subsubsection{How Spammers Operate}

This subsection discusses the means by which spammers operate, including: methods for acquiring email addresses to spam, techniques they deploy to deliver spam messages, and tricks they employ to confuse and bypass some spam filters.

\subsubsection{Gathering of Email Addresses}

Spammers first need to obtain the email addresses of intended recipients before sending out mass junk mail. For this, the spammers themselves and junk mail merchants compile huge lists of prospective (but not guaranteed valid) email addresses. This action is usually done without consent of the individuals whose emails addresses have been collected.

Email harvesting is the process of obtaining lists of email addresses for use in bulk email or other purposes usually grouped as spam [60]. As discussed in [41], below are some common methods of obtaining email addresses.

1. Using a special automated software, a.k.a. "email harvesters" or "harvesting bots" which wanders around the web, scanning pages, newsgroups and online forums searching for username@domain.com like addresses. Spammers are able to gather thousands of email addresses with this approach, and they could use a separate program to test each address for validity.

2. Purchasing lists of addresses from other spammers, or persons looking to profit from valid lists. These lists may include millions of email addresses. Spammers simply load their lists into bulk mail programs and execute simple commands to distribute mass emails.

3. Using deceptive measures to trick users into giving up their email address. For example, using swindle websites to attract people to enter into an appealing competition. The deception here is that users must enter their email address to compete, and then they are on another spam list.

4. Using dictionary attack spamming - a sophisticated guesswork to generate addresses using people's names, numbers, known email addresses, and other known 
facts. This method can expose email addresses that have never even been published on the internet.

\subsubsection{Delivery of Spam Messages}

Provided with an internet connection, a mailing address list, a mass mailing software program, and a profitable idea to be relayed in a message, a spammer is ready to go to work delivering spam messages. They employ several techniques to gain access to email servers used to deliver all email to their destination addresses. Listed below are some frequently used spam sending techniques.

1. Open proxy servers are the most widely used method of sending spam today. A proxy server is a server used within a network that other computers use as a gateway to the internet. Products such as Wingate, Squid, and ISA servers are commonly used proxies [73]. An open proxy is a proxy server which is set up to accept client connections from any IP address and make connections to any internet resource. Spammers frequently install open proxies on unwitting end users' operating systems by means of computer viruses designed for this purpose. This is a problem because the open proxy server hides the source IP of the spammer, by establishing all connections on their behalf to avoid detection.

2. An open mail relay is an SMTP (email) server configured in such a way that it allows anyone on the Internet to send email through it. Spammers take advantage of open relays to re-route their email through third party email servers to avoid detection.

3. Botnets. Easy detection of using either a proxy server or an SMTP relay to send spam emails has led spammers to become extra innovative, collaborating with hackers, virus and worm creators, to create spamming networks called botnets [61]. These networks are armies of compromised machines known as zombies, which are controlled by a single master. Zombies, once controlled will do anything including send spam, and they are highly configurable and easy 
to maintain. Having control over thousands of hosts that are not known open proxy servers is perfect for spammers, as they get exclusive access to use this host as if it was their own [73].

\subsubsection{Exploits in Spam}

Today there are a large number of techniques employed to help eliminate spam, including spam filtering. As such spammers have had to employ various techniques to avoid being detected by spam filters. Some spammer exploits were clearly presented in a paper by John Graham-Cumming, titled "The Spammers' Compendium" [26]. Staff of Microsoft Corporation analyzed a large junk email corpus generated by volunteer users of the Hotmail email service to determine what type of product spammers promote, as well as the exploits they use to avoid detection [32]. Table 2.2 shows the specific exploits presented as a result. 


\begin{tabular}{|l|l|}
\hline Exploit & Description \\
\hline Word Obscuring & Misspelling words, putting words into images, etc. \\
URL Spamming & Adding URLs to non-spam sites (e.g. msn.com). \\
Domain Spoofing & Using an invalid or fake domain in the from line. \\
Token Breaking & Breaking words with punctuation, space, etc. \\
MIME Attacks & Putting non-spam content in one body part and spam content in another. \\
Text Chaff & Random strings of characters, random series or words, or unrelated sentences. \\
URL Obscuring & Encoding a URL in hexadecimal, hiding the true URL with an @ sign, etc. \\
Character Encoding & Phar\&\#109;acy renders into Pharmacy. \\
\hline
\end{tabular}

Table 2.2: Exploits in spam

\subsubsection{The Issues, Impact and Cost of Spam}

For many individuals it is mainly a nuisance to receive huge amounts of unwanted messages; it wastes time to read, filter through, and delete or even respond to spam emails. Through spam, users are exposed to possible fraudulent exploits, like identity theft, and without good security practices their personal computers are exposed to malicious code: viruses, Trojan horses and worms, amongst others. Additionally, the sexually explicit nature of many spam messages is offensive and concerning to many email users.

For most organizations spam is more than just an irritation; given that as spam volumes rise, the value of email as a business tool within the corporate world diminishes. Spam generally impacts organizations' productivity and operational costs, as well as exposes them to many risks. It has been identified that spam negatively impacts organizations in these universal ways: reduced employee productivity and security, increase in network resource costs, IT administration costs and legal liability risk [5].

First, consider the impact to employee productivity. Large organizations lose productivity to the spam problem as employees on the whole dedicate a considerable part of their work time dealing with spam emails. As estimated in [5], a large organization with 52,000 employees would lose $\$ 14$ million. Note that cost estimates vary amongst many market researchers. 
Reduced security is another impact of spam. Spam also poses security risks including identity theft, fraud, viruses, worms, and malicious code. Without good security practices, end users computers are compromised, and even outsourcing spam solutions require organizations to hand over control of their confidential information to a third party.

Thirdly, consider the increased cost for networking infrastructure: software, hardware, storage and bandwidth which are needed to process and store the high volume of emails consisting of a high proportion of spam. Storage resources and processing cycles consumed by this high volume of emails unswervingly impact network productivity. If half of incoming emails are categorized as spam, then half of networking resources, a representation of at least $50 \%$ of network costs will be dedicated to facilitate spam emails to improve overall system performance. A legal firm shared findings of their spam costs with Network World [19], and some claims were that spam volumes consumed bandwidth accounting for an extra $\$ 17,000$, and that storage costs were pegged at $\$ 114,000$ more.

Now, factor in the impact to IT administration costs. If additional networking infrastructure is required to deal with the problem of spam, more IT staff is also required to effectively manage systems and to assist end users. This includes technical, helpdesk and systems administration personnel.

Last but not least; consider the risk of legal accountability, as some spam offensive messages may create a hostile work environment [5]. Notwithstanding warnings and policies some employees may still exchange racist, pornographical, hate inciting and other offensive messages that anger some colleagues who may take legal action.

\subsubsection{Phishing and Spam Scams}

Spam has become a widely used method of advertising as the same message can be sent out to millions of mailboxes with little overhead. See figure 2.4 at the end of this section for an example of an advertising spam message. Some recipients of advertising spam find it informative while others find it annoying and time consuming. Besides those spammers who only seek to advertise their products, there exist those who seek to exploit naive users by luring them to trust bogus offers and fraudulent 
claims that will only lead to loss of money or identity theft. The spammer's goal here is to defraud recipients and then disappear. A particularly dangerous and common type of spam is phishing, an attempt to defraud people of their personal information including credit card or banking information, social security numbers, and more. In this subsection, I will discuss phishing and spam scams.

\subsubsection{Phishing}

As discussed in a white paper by McAfee [81], phishing attacks use both social engineering and technical artifice to steal an individual's personal identity data and financial account credentials. McAfee describes social engineering schemes to use spoofed emails to lead recipients to counterfeit Web sites designed to trick them into disclosing their financial data, by hijacking brand names of banks, e-retailers, and credit card companies to elicit a response. For example, figure 2.3 at the end of this section shows a PayPal phishing scam, where clicking on the provided link (https://www.paypal.com/cgi-bin/webscr?cmd=resolution-center) redirects to a web page designed identical to PayPal; not the actual PayPal page. Technical artifice schemes, on the other hand, plant crimeware onto PCs using Trojan keylogger spyware, in order to steal credentials directly.

Drake, etc. in their paper titled "The Anatomy of a Phishing Email" [14], explore numerous tricks used in phishing emails. Below are some samples taken from their analysis of tricks used in phishing emails as employed in phishing emails forwarded to MailFrontier from its customers:

\section{Attempt to Spoof Reputable Companies}

In order to convince recipients to give up their personal information, phishers must gain their trust. They do this by using convincing strategies like trying to spoof or mimic reputable companies like eBay, PayPal, Citibank etc. Some strategies include:

- Using the legitimate company's logo, similar font and color schemes as used on the company's web site.

- Providing links to sections of the legitimate websites/services 
- Spoofing "from" email address to make it appear to be from some reputable company (e.g. eBay.com, paypal.com etc).

\section{Reply Address Differs from the Claimed Reputable Sender}

In some cases, the email claims to be sent from a reputable company, however, the reply address is a fraudulent one. For example an email claiming to be sent from Citibank may have a fraudulent reply address like citibank@ecitibanking.com

\section{Creating a Convincing Premise}

Some fraudulent emails try to convince the recipient to reveal personal information by making false claims like: their account information is outdated, a credit card is expired, their account has been randomly selected for verification, etc.

\section{Links to Seemingly Reputable Web Sites}

Today, most phishing emails provide a link to a website for a domain they have registered with a name similar to a reputable company. Usually the lirik seen in the email is different from the actual link destination. The Web site for this link is designed to look exactly like that of the reputable company, tricking the recipient into assuming nothing of a fraudulent activity. Phishers may do this by using the JavaScript event handler "onMouseOver" to show a false URL in the status bar of the user's email application, or by using HTML code to display a false link which only directs users to fraudulent Web sites.

\subsubsection{Spam Scams}

Some email users have lost money to spam containing false offers and deceptive promotions. OnGuardOnline.gov provides practical tips from the U.S federal government and the technology industry to help guard against internet fraud, secure computers, and protect your personal information. In an article they published [66], 10 email scams to screen are discussed namely: Phishing, The "Nigerian" Scam, Work-at-Home Scams, Weight Loss Claims, Foreign Lotteries, Cure-All Products, Check Overpayment Scams, Pay-in-Advance Credit Offers, Debt Relief, and Investment Schemes. Below I will present four of these scams as they were discussed: 


\section{1. "Nigerian" Scam}

In this scam the con artists usually claim to be officials, business people, or the surviving spouses of a past government official in some country whose money is somehow tied up. Nigeria is a commonly used country although other countries are also mentioned. The email tries to convince the recipient to help them access their money, by offering to transfer a lot of money into their bank accounts if they agree to send money to cover some fees needed to be paid before the release of this tied up money. Agreeing to help possibly means sending them money, most likely revealing your bank information, or other information. Ultimately, there are no returns for the recipient; rather the scam artist vanishes with your money.

\section{Weight Loss Gimmicks}

These are emails advertising weight loss products like pills, creams, patches, amongst others. They warrant their products to have highly effective results without the need for dieting or exercise. Being a scam, these products will never be delivered after receiving payment.

\section{Work From Home Scams}

This scheme aims to convince recipients (via advertising) of a means of securing a steady income with minimal work from home. Their ads include medical claims processing, envelope-stuffing, craft assembly work, or other jobs. They however don't disclose much information regarding hours of work, hidden costs of ads to be paid by the recipients, operational costs, and more. Once the recipient commits time and money, there is also the likelihood that some promoters may refuse to pay with bogus claims.

\section{Pre-Approved Credit Offers}

This scam targets people with bad credit by offering them a pre-qualified low interest credit card or loan, if they are willing to pay a processing fee. There is actually no loan so compliance will result in a loss of processing fees to scam artists. 


\section{PEApPa $]^{\circ}$}

Paypal is constantly working to ensure security by regularly screening the accounts in our system. We recently reviewed your account, and we need more information to help us provide you with secure service. Until we can collect this information, your access to sensitive account features will be limited. We would like to restore your access as soon as possible, and we apologize for the inconvenience.

Why is my account access limited?

Your account access has been limited for the following reason(s):

April, 2007: We have reason to believe that your account was accessed by a third party. Because protecting the security of your account is our primary concern, we have limited access to sensitive Paypal account features. We understand that this may be an inconvenience but please understand that this temporary limitation is for your protection.

(Your case ID for this reason is PP-257-057-154.)

To remove the limitation click on the following link:

https://www.paypal.com/cgi-bin/webscr?cmd= resolution-center

\section{Regards,}

\section{PayPal Security Departament}

Copyright 62007 Paypal Inc. All rights reserved. Designated trademarks and brands are the property of their respective owners. PayPal is located at 2211 N. First St., San Jose, CA 95131.

Figure 2.3: An example of a PayPal phishing scam. This is not an obvious spam; however investigations revealed that the seemingly reputable link displayed (https://www.paypal.com/cgi$\mathrm{bin} /$ webscr? $\mathrm{cmd}=$ resolution-center) is different from the actual link destination. 


\section{Reduce Your Debt Today!}

\section{Take a moment to see how our expert} debt negotiators can help you reduce your debt to a fraction of what it is today!

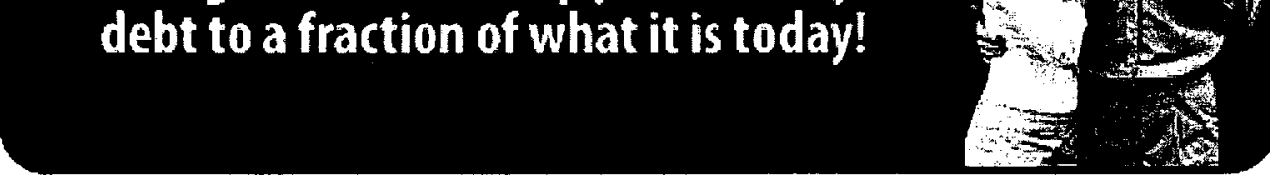

Do you feel trapped under a mountain of debt?

Having large amounts of debt can be more than a small headache. Our expert debt negotiators can help you get your finances back on the right track by reducing your current debt to a fraction of what it is today.

Take just a moment and get your TOTALLY FREE, ZERO OBLIGATION debt analysis today!
- Stop Creditor Phone Calls

- Reduce Monthly Payments

- Repay As Little As 40-60\%

- Become Totally Debt Free

\section{Get Started} Today

\section{CLICK HERE FOR YOUR FREE DEBT ANALYSIS}

Figure 2.4: An example of spam message advertising debt reduction 


\subsection{Solutions for Combating Spam}

There are a number of solutions available to combat email spam. Many of these solutions have encouraged my work, however the experiments presented in this thesis were most influenced by supervised learning algorithms in the context of statistical spam filtering. Described below are: legislative and legal spam actions, technical initiatives to fight spam including: black and white listing, reverse DNS lookup, content and statistical spam filtering techniques.

\subsubsection{Legislative and Legal Action}

Legislative and legal action is a solution employed by individual countries to combat spam. Although Canada is currently examining the need for spam-specific legislation, it is technically not against the law to send spam [33]. The U.S. on the other hand have the CAN-SPAM Act of 2003 [75], which establishes requirements for those who send commercial email, spelling out penalties for spammers and companies whose products are advertised in spam if they violate the law.

Legislation has had little impact in curbing the spam issue as evidenced by the high volumes of spam still being received. According to an analysis of the effect of international spam legislation by Vircom Inc. [8], for spam to ever be effectively controlled by legislation, several considerations must be made. These will include actually enforcing the legislation with penalties for the crime, implementing a worldwide legislative approach to avoid spamming from countries with weaker or no spam laws, increasing public knowledge about the issue, and combining the approach of legislation with technology.

\subsubsection{Black and White Listing}

Black and white lists are the most basic forms of blocking spam. Databases are maintained with lists of known untrustworthy spam sources (blacklists) or with lists of known legitimate email sources (whitelists). Entries in these lists could be a single IP address, email address or an entire domain. These lists should be frequently updated so that a filter using them is also effectively updated. 
Using updated lists, any email originating from a blacklisted source will be quickly blocked from reaching its intended destination. The opposite action occurs with whitelists, which allow only members of that list. Accepting email sent from white listed servers without further filtering can be a highly effective way to reduce false positives resulting from aggressive spam controlling measures [71].

Management of these lists may be at a domain-level by a domain administrator or by ESPs/ISPs where distributed lists connect to an MTA as an access controlling mechanism, to allow or disallow delivery of a message to intended recipients.

\subsubsection{Reverse DNS Lookup}

DNS [1] forward lookup is used to determine the associated IP address for a given hostname; conversely a reversely lookup is used to reverse resolve the associated hostname for a given IP address. Many mail servers will refuse email with missing reverse DNS names.

Given this capability, another email anti-spam technique is to check the domain names in reverse DNS to see if they belong to spammers, and if so may be excluded from an email user's legitimate emails [1].

Reverse DNS lookups, although popular can generate a high false positive (emails claimed to be spam when actually legitimate) rate, since every single existent domain name is not likely to have a properly established reverse DNS lookup, causing emails from some legitimate domains to be rejected.

\subsubsection{Content Filtering}

Content filtering is a simple yet effective way to block a majority of obvious spam. Emails are sorted based on content specific information found in particular header fields, the general header, and/or the body of an email message. Content filters identify emails containing certain keywords, phrases or character combinations known to indicate spam. Spam is generally repetitive and in most cases will contain some common features. There are different types of content filters that vary based on the features they are designed to detect. 
Word based spam filters are the simplest form. They trace certain words within the email and block it. It is possible to evade these types of spam filters by configuring the message to remove the most common words in spam by either replacing them or misspelling these words.

Heuristic filters are more complex than word filters, used to trace multiple terms found in an email. They are also called rule filters or rule-based scoring [73]. Their operation involves scanning the contents of incoming emails and assigning points to certain known words or phrases. The flexibility of pattern matching techniques, like regular expressions [20] extends the capabilities of content filtering by describing an email in terms of more complex patterns by examining the header, layout, overall organization, and other characteristics typical of spam. Rules are formulated to analyze and filter incoming email messages. A matched rule may increase or decrease the overall score for an individual email message. Rules matching keywords found in spam receive higher points and a total score for the entire email is determined. For a given email, if the spam score reaches a certain threshold, then the filter classifies it as spam thus blocking or deleting it. System administrators determine the cut off score that will be used to classify email as spam or legitimate. Heuristic filters are relatively easy to operate and quite fast.

A major disadvantage of content filters is that they can filter off legitimate email as well if it happens to contain a high usage of certain words or phrases. Alternatively, mail spammers may avoid them by avoiding the use of these words in their email messages. Also, content filtering is time consuming to administrators who are constantly updating rules to keep up with steady evolving spam traits [73]. Also, this approach to filtering is prone to false positives as the system may reject legitimate messages on the assumption that they are spam. A rule attempting to reject spam messages may unintentionally block legitimate emails having similar keywords found in spam. Next we will examine a statistical content analysis as a better solution to content heuristics. 


\subsubsection{Statistical Filtering}

Although some server-level spam filters are heuristic based; a wide number of them utilize statistical filtering $[97,73]$, which by far is the most effective spam fighting technique. They have evolved over the last few years to be a popular highly effective spam filtering mechanism that is applied by the majority of the email clients to sort mail into junk (spam) or inbox (legitimate) folders. Statistical filters are modeled from a collection of emails (corpus), using supervised machine learning algorithms. There are various algorithms that are currently applied in the fight against spam including - Naive Bayes, Maximum Entropy Model, Memory-Based Learning, Support Vector Machine and Boosting [98].

Statistical algorithms measure the likelihood that an email message is spam based on the prior occurrence of certain elements ("tokens") in pre-classified documents. They analyze emails to monitor unique differences in the message by inspecting tokens like words/phrases, transport routes in message headers, and other entities like HTML code (like fonts), images and attachments [78]. Based on this prior analysis, they can resolve the likelihood that an email found to contain certain known tokens is considered as spam. A statistical filter will also adjust the settings in the content of spam emails as soon as it can detect changes.

Bayesian filters are currently the most popular statistical content filters. They evaluate the mathematical probability that a message is spam or legitimate based on features that are evident in a collection of emails. These filters have a set of word tokens which are assigned specific statistical values in a database [12]. The filter acquires words from email messages and compiles them it to a list which it applies to block or delete spam. The Bayesian algorithm however loses efficiency with time as it increases the word list.

Bayesian filters are included in versions of SpamAssassin, Anti-Spam SMTP Proxy (ASSP) and SpamBayes. Other software programs that use the Bayesian classification include Bogofilter, Mozilla thunderbird and Mailwasher. CRM114 is a recent innovation that detects spam by Bayesian classification on the phrases within the messages. It is possible to install the functionality of these programs within the main server software that takes care of a greater number of mails. Once it is installed and 
given directives, spam filtering software requires no other maintenance follow ups. The user is only required to mark messages as spam or non spam and the filter will learn from the user's ruling of the content in email messages [44].

Statistical filters account for low rate of false positives, require less manual intervention, have the ability to be personalized to individual user decisions, and are able to automatically adapt to changes in spam. Even though statistical filtering has been very successful in its ability to filter spam, some evasion techniques have been identified [32]. Consequently, new approaches are desired to deal with the persistent attempts by spammers to break the existing anti-spam barriers. Inspired by statistical spam methods, this thesis presents a rough set/VPRSM approach to spam filter learning. 


\section{Chapter 3}

\section{Theoretical Aspects of Research}

This chapter focuses on the theoretical aspects of the research, specifically the application of machine learning $[7,42,16,3]$ to extract generalized patterns/rules from large data sets, as relating to the spam detection application presented in chapter 4 . Section 3.1 provides an overview of a categorization technique, the original rough set theory as proposed by Zdzislaw I. Pawlak $[57,54,52,49,51]$ in the early 1980s. In section 3.2 , the focus shifts to the probabilistic extension of the original theory, the variable precision rough set model VPRSM - the technique used to model the spam detection system presented in this thesis.

\subsection{Overview of Rough Set Theory}

Pawlak proposed the rough set theory $[57,50,55,56,69]$ as a formal framework for the automated transformation of data into knowledge. Given a collection of data objects (emails in our case) from a universe of interest, a knowledge representation system (KRS) is employed to express observations about the objects collected. A decision table is a type of KRS that represents a relation, typically functional or partially functional, between a group of input values and a set of output values, known as condition and decision attributes respectively [106, 10, 57, 93]. Decision tables are learned or generated from the collection of data objects. Using the concept of rough sets, one can extract a generalized description of objects contained in such decision tables. Extracted descriptions in the form of rules are utilized to sort new objects (emails). The subsections that follow will discuss the basic concepts of the 
rough set theory, as well as the concept of set approximations.

The rough set theory is a mathematical approach applicable to machine learning, particularly useful for training controlled systems, development of data-based decision support systems and pattern recognition [113]. Related work on the application of this theory has been reported by many $[86,85,74,68]$.

\subsubsection{Basic Concepts}

Let $K$ be a KRS such that $K=(U, A)$, where $U$ is a non-empty finite subset of objects from a universe of interest, and $A$ is referred to as attributes expressing observations acquired from objects in $U[57,112]$. In other words, $\mathrm{K}$ can be viewed as a system that maps each attribute $a \in A$ to a value in $V_{a}$, for every object in a defined universe. This mapping is denoted $a: U \rightarrow V_{a}$, where $V_{a}$ is a finite set of values called the domain of the attribute $a[110]$.

Now consider a decision table $T=(U, A, C, D)[57,59]$ to be an important class of knowledge representation systems, with distinguished subsets of attributes namely $C$ and $D$, such that $C, D \subset A[57]$. Elements belonging to set $C$ are variables referred to as condition attributes (inputs), whereas the variables in $D$ are called decision attributes (outputs) $[116,85,57,112,70]$. The observed values of decision attributes typically do change in response to manipulated values of condition attributes. In short, decision tables are used to capture complicated logic of condition/decision attribute relationships into a compact tabular view, processed to generate conditional statements or expressions. These statements associate conditionals or inputs (subsets of C) with corresponding decisions or outputs. Decision tables are an efficient approach to describe problems where unstable conditions produce different outcomes.

\section{Example 1: Decision Table}

Say that auto insurance applications were processed based on three determining factors, each of which has two possible outcomes, yes $(\mathrm{Y})$ or no(N). The first factor is to ensure that the applicant has not exceeded the threshold for the maximum number of accidents (i.e. $C_{1}=$ Has many accidents?). The second factor is based on the type of car - the more expensive your car, the more you will pay. (i.e. $C_{2}=$ Is type 
expensive?). The third factor is the applicants age - drivers under the age of 25 are charged higher rates. (i.e. $C_{3}=$ Is age $<25$ ?).

Applications are submitted electronically and the insurance company writes the decision table below to automatically label applications for further processing. Available labels are: $\mathrm{D}=\{1$-Criteria not met, 2-Standard criteria met, 3-Special criteria met $\}$ :

\begin{tabular}{|c|c|c||c|}
\hline$C_{1}$ & $C_{2}$ & $C_{3}$ & $D$ \\
\hline$Y$ & $Y$ & $Y$ & 1 \\
$Y$ & $Y$ & $N$ & 1 \\
$Y$ & $N$ & $N$ & 1 \\
$N$ & $N$ & $N$ & 2 \\
$N$ & $Y$ & $N$ & 3 \\
$N$ & $N$ & $Y$ & 3 \\
$\ldots$ & $\ldots$ & $\ldots$ & $\ldots$ \\
\hline
\end{tabular}

Next, we discuss the concept of an equivalence relation. An equivalence relation $R$ of a set $U$ represents the classification of $U$ that identifies subgroups in $U$, each of which contains elements that are alike in some way [57]. The relation $\mathrm{R}$ is reflexive, symmetric, and transitive [96, 94, 95]. In other words, if $R$ is an equivalence relation over $U$, then $U / R$ denotes the family of all classes of the classification of $U$. Every individual class of the classification of $U$ containing an element $x \in U$ is denoted as $[x]_{R}$. The family of all equivalence relations over $U$ conceptually represents the knowledge about the universe of interest. Equivalence classes induced by $R$ are also called elementary sets in $K[53,30]$.

With that said on knowledge bases and equivalence relations, now let $K$ represent a knowledge base such that $K=(U, A)$. Then for any subset of attributes $P \subseteq A$ such that $P \neq \emptyset$, there exists an associated equivalence relation called the indiscernibility relation over $P$, denoted by $I N D(P)[100,92,10,82]$. If two objects $x, y \in I N D(P)$, it implies that both objects are indiscernible using attributes of the relation $P$, such that $\forall q \in P: q(x)=q(y)$. That is, two objects are indiscernible over a relation $P$ if they have the same values across all attributes of $P$. 


\section{Example 2: Knowledge base and equivalence classes}

$U=\left\{x_{1}, x_{2}, x_{3}, x_{4}, x_{5}, x_{6}, x_{7}, x_{8}\right\}$

$A=\left\{a_{1}, a_{2}, a_{3}, a_{4}, a_{5}, a_{6}\right\}$

Given $(U, A)$, we can express the different classifications of $U$, according to an equivalence relation $P \subseteq A$ over $U$. If we select $P=A$ (i.e $\mathrm{P}$ is the full set of attributes), we can represent the objects $x \in U$ in terms of attributes and their values as:

\begin{tabular}{|c||c|c|c|c|c|c|}
\hline$U$ & $a_{1}$ & $a_{2}$ & $a_{3}$ & $a_{4}$ & $a_{5}$ & $a_{6}$ \\
\hline$x_{1}$ & 1 & 1 & 1 & 1 & 1 & 1 \\
$x_{2}$ & 1 & 1 & 1 & 1 & 1 & 1 \\
$x_{3}$ & 1 & 1 & 1 & 1 & 1 & 0 \\
$x_{4}$ & 1 & 1 & 1 & 1 & 1 & 1 \\
$x_{5}$ & 1 & 1 & 1 & 1 & 0 & 0 \\
$x_{6}$ & 1 & 0 & 0 & 0 & 0 & 1 \\
$x_{7}$ & 0 & 0 & 0 & 1 & 1 & 1 \\
$x_{8}$ & 0 & 0 & 0 & 0 & 0 & 0 \\
\hline
\end{tabular}

Given $P=A=\left\{a_{1}, a_{2}, a_{3}, a_{4}, a_{5}, a_{6}\right\}$ as illustrated above, we can deduce the following equivalence classes: $\left\{x_{1}, x_{2}, x_{4}\right\},\left\{x_{3}\right\},\left\{x_{5}\right\},\left\{x_{6}\right\},\left\{x_{7}\right\}$ and $\left\{x_{8}\right\}$. However, if $P \subset A$ such that $P=\left\{a_{1}, a_{2}, a_{3}\right\}$ we can deduce the following equivalence classes: $\left\{x_{1}, x_{2}, x_{3}, x_{4}, x_{5}\right\},\left\{x_{6}\right\}$ and $\left\{x_{7}, x_{8}\right\}$.

\subsubsection{Set Approximations}

Concepts described in the previous section can be applied to approximately induce categorical partitions of a universe $U$. In particular, given distinguished condition $C$ and decision $D$ attributes of a decision table $T$, the respective partitions $U / C$ and $U / D$ exist $[57,55]$. The partition $U / C$ will represent tuples expressing how properties (values of condition attributes) of individual objects relate or differ from 
one another. Partition $U / D$ expresses classes of $U$ as corresponding to different values of the decision attribue. The pair $(U, U / C)$ is referred to as an approximation space, the $C$-elementary sets $E \in U / C$ are referred to as elementary sets, and the $D$-elementary sets $F \in U / D$ will be called decision categories $[22,110,56]$.

Pawlak proposed an idea of approximating a set (say target set $X$ ) by other sets via the theory of rough sets $[57,54,52,51]$. In particular the theory of rough sets can be used to express relations between condition and decision attributes, as represented by the partitions of $U$ [57]. The rough set theory is made up of two basic set concepts - the upper and lower approximations of a set $X$ in question $[57,55,79,89,69]$. Specifically, given knowledge base $K=(U, P), P \subseteq A$ then the set $X \subseteq U / D$ can be approximated by forming the lower and upper approximations of $X$.

The lower approximation $\underline{P} X$, includes those objects contained in $U$, which can with certainty be classified as elements of the set $X$ using knowledge base $K$, and is defined as:

$$
\underline{P} X=\bigcup\{Y \in U / P: Y \subseteq X\}
$$

The upper approximation $\bar{P} X$, includes those objects contained in $U$, which can possibly be classified as elements of $X$ using knowledge base $K$, defined as:

$$
\bar{P} X=\bigcup\{Y \in U / P: Y \cap X \neq \emptyset\}
$$

The lower and upper approximations can also be represented in the equivalent forms shown below, using $[x]_{P}$, the equivalence class of $P$ containing $x \in U$ :

$$
\begin{gathered}
P X=\left\{x \in U:[x]_{P} \subseteq X\right\} \\
\bar{P} X=\left\{x \in U:[x]_{P} \cap X \neq \emptyset\right\}
\end{gathered}
$$

To satisfy the initial interest of expressing relations between condition and decision attributes, upper and lower approximations of the target set $X$, are used to divide the universe into three disjoint regions - positive, negative and boundary regions of $X$ [57]. The positive region $P O S_{P}(X)$ is basically calculated as the lower approximation 
of the target set, and translates to those objects in $U$ whose condition attribute value map to a decision category corresponding to $X$. The negative region $N E G_{P}(X)$, consists of those objects that can with certainty be classified as not belonging to $X$, employing the available knowledge $P$. Such objects belonging to $\neg X$ thus belong to the complement set of $X$ expressed as $\neg X$. The boundary region $B N D_{P}(X)$, is the non-certain area of the universe, and consists of those objects belonging to the borderline which cannot be classified into either set $X$ or $\neg X$, at least using knowledge base $K$.

$$
\begin{gathered}
P O S_{P}(X)=\underline{P} X \\
N E G_{P}(X)=U-\bar{P} X \\
B N D_{P}(X)=\bar{P} X-\underline{P} X
\end{gathered}
$$

The presence of $B N D_{P}(X)$ in the approximation of set $X$ informs us that it is a rough set, such that $\bar{P} X-\underline{P} X \neq \emptyset$; otherwise $X$ is described as crisp such that $\bar{P} X-\underline{P} X=\emptyset[57]$. The variance between the roughness and crispness of the approximations of a set $X$ represents the accuracy of approximation. Intuitively, the more objects we have falling into the boundary region of an approximation will lower the accuracy of a set's approximation. The accuracy measure $0 \leq \alpha(X) \leq 1$ for set approximation is defined as:

$$
\alpha(X)=\frac{|\underline{P} X|}{|\bar{P} X|}
$$

If $\alpha(X)=0$, then the extent of roughness is reached, meaning that the set $X$ is undefinable w.r.t $P$ and $B N D_{P}(X) \neq \emptyset$. Also, $\alpha(X)=1$ implies a perfect approximation i.e crisp, meaning that the set $X$ is definable w.r.t $P$ and $B N D_{P}(X)=\emptyset$, so every element in $X$ can be classified using knowledge $P$.

\section{Example 3: Approximating a circle using rough sets}




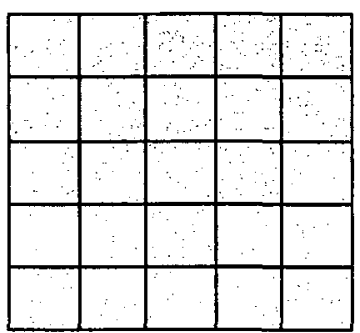

Approximation Space

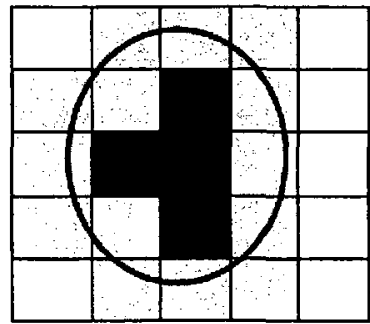

Positive Region (black)

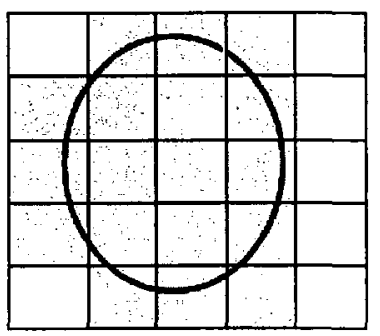

Negative Region (white)

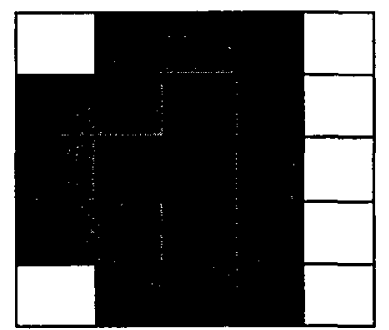

Boundary Region (dark grey)

Figure 3.1: Approximation of a circle using rough sets

Figure 3.1 illustrates the approximation of a set bounded by an oval using rough sets. From left to right, top to bottom: the approximation space; the negative region marked in white; the positive region marked in black; boundary region (the actual approximation) marked in dark grey [10]. 


\section{Example 4: Approximating a target set $X$ using rough sets}

Given the knowledge base $K=(U, P)$ from example 2, we can define a decision table $T=(U, P, C, D)$ such that $C=\left\{a_{1}, a_{2}, a_{3}, a_{4}, a_{5},\right\}$ and $D=\left\{a_{6}\right\}:$

\begin{tabular}{|c||c|c|c|c|c|c|}
\hline$U$ & $a_{1}$ & $a_{2}$ & $a_{3}$ & $a_{4}$ & $a_{5}$ & $a_{6}$ \\
\hline$x_{1}$ & 1 & 1 & 1 & 1 & 1 & 1 \\
$x_{2}$ & 1 & 1 & 1 & 1 & 1 & 1 \\
$x_{3}$ & 1 & 1 & 1 & 1 & 1 & 0 \\
$x_{4}$ & 1 & 1 & 1 & 1 & 1 & 1 \\
$x_{5}$ & 1 & 1 & 1 & 1 & 0 & 0 \\
$x_{6}$ & 1 & 0 & 0 & 0 & 0 & 1 \\
$x_{7}$ & 0 & 0 & 0 & 1 & 1 & 1 \\
$x_{8}$ & 0 & 0 & 0 & 0 & 0 & 0 \\
\hline
\end{tabular}

Assume here that the set of all objects in $U$ can be classified as belonging to either the category 0 or category 1 , depending on the relative outcome of the corresponding binary decision attribute.

Let the target set $X$ represent those objects which can certainly be classified as belonging to category 1 based on knowledge $P$. Then the lower and upper approximations of $X$ are:

$$
\begin{aligned}
& \underline{P} X=\left\{x_{6}, x_{7}\right\} \\
& \bar{P} X=\left\{x_{1}, x_{2}, x_{3}, x_{4}, x_{6}, x_{7}\right\}
\end{aligned}
$$

As expressed in $T$, the lower approximation of $X$ represents all objects in $U$ with a decision attribute value corresponding to the target set in question (i.e. category 1), such that there exists no other objects in $U$ with identical condition attribute values but a conflicting decision attribute value corresponding to to the target set in question. On the other hand, the upper approximation represents the lower approximation plus all objects in $U$ with identical condition attribute values, but with 
conflicting decision attribute values such that the decision value for at least one of these objects corresponds to the target set in question.

Further, the upper and lower approximations of the target set $X$, can be used to divide $U$ into three disjoint regions as:

$$
\begin{aligned}
& P O S_{P}(X)=\underline{P} X=\left\{x_{6}, x_{7}\right\} \\
& N E G_{P}(X)=U-\bar{P} X=\left\{x_{5}, x_{8}\right\} \\
& B N D_{P}(X)=\bar{P} X-\underline{P} X=\left\{x_{1}, x_{2}, x_{3}, x_{4}\right\}
\end{aligned}
$$

\subsubsection{Rough Sets for Classification}

The concept of set approximations can be directly applied to the task of classifying objects according to their true categories - for example to classify email documents as spam or legitimate.

Consider a binary classification problem, where objects of a universe $U$ may be classified as either belonging to a target set $X$, or it's complementary set $\neg X$. To that end, $X$ may be approximated using rough sets to arrive at three approximate regions for $U$. Decision rules $[40,2,67,87,102,104]$ can then be extracted from each of these regions and used to classify new cases. Literally, objects matching a rule obtained from $P O S(X)$ regions will conclude those objects as certainly belonging to category $X$. The $N E G(X)$ region rules will classify matching objects as certain members of category $\neg X$. Any $B N D(X)$ region rules are unable to certainly classify matching objects as members of $X$ or $\neg X$.

Approximate regions are formed in a defined decision table, implying that rules can be extracted from every unique tuple as logical implications that map a set of condition attribute values to a decision value, for a given knowledge representation. For simplicity here, we may assume that much cannot can be said about objects falling into the $B N D(X)$ region so we ignore their associated rules (Such cases will be addressed in section 3.2.4). A classifier can be built using rules obtained from 
decision tables to classify objects as $X$ or $\neg X$.

\section{Example 5: Rule formation}

Given the task to classify email documents as either spam or legitimate (non-spam), using the decision table defined in example 4. Assume that the approximation presented was based on the target set $X$ being spam, where the binary decision attribute $D$ implies object category - spam with a decision value 1 , otherwise legitimate with a decision value 0 .

For email document classification we can extract rules from $P O S_{P}(X)$ and $N E G_{P}(X)$ regions, to imply the category spam or legitimate respectively. From $\operatorname{POS}_{P}(X)$ region, extract rules from corresponding objects (i.e. $\left\{x_{6}, x_{7}\right\}$ ) represented in logical form below. Such rules classify any new object as spam, if that object's conditional attribute values match those of the rule itself.

$$
\begin{aligned}
& \left(a_{1}=1\right) \wedge\left(a_{2}=0\right) \wedge\left(a_{3}=0\right) \wedge\left(a_{4}=0\right) \wedge\left(a_{5}=0\right) \rightarrow \text { spam } \\
& \left(a_{1}=0\right) \wedge\left(a_{2}=0\right) \wedge\left(a_{3}=0\right) \wedge\left(a_{4}=1\right) \wedge\left(a_{5}=1\right) \rightarrow \text { spam }
\end{aligned}
$$

Similarly, extract rules from all objects falling in $N E G_{P}(X)$ region (i.e. $\left\{x_{5}, x_{6}, x_{7}, x_{8}\right\}$ ). This set of rules classify any new object as legitimate, if that object's conditional attribute values match those of the rule itself.

$$
\begin{aligned}
& \left(a_{1}=1\right) \wedge\left(a_{2}=1\right) \wedge\left(a_{3}=1\right) \wedge\left(a_{4}=0\right) \wedge\left(a_{5}=0\right) \rightarrow \text { legitimate } \\
& \left(a_{1}=0\right) \wedge\left(a_{2}=0\right) \wedge\left(a_{3}=0\right) \wedge\left(a_{4}=0\right) \wedge\left(a_{5}=0\right) \rightarrow \text { legitimate }
\end{aligned}
$$


Rough sets can also determine the minimum number of attributes needed for classification. This is directly achieved via attribute dimensionality reduction $[10,11]$ an important technique for reducing irrelevant concepts presented by the knowledge represented, and in turn shortens the rules extracted. Several classification tasks deal with large domain knowledge that adversely affect accuracy or computational time, however not all information contained is necessary so it is worth reducing redundant attributes. So the question then is how do we determine which attributes are irrelevant? Those irrelevant attributes are addressed by two fundamental concepts to rough set attribute reduction known as reduct and core $[10,36,57]$.

A reduct can be described as a minimal subset of attributes that is sufficient to preserve the equivalence structure of the universe, given an information system $I=(U, A)$ like that presented in example 2 . In other words, a reduct is a minimal set of attributes $B \subseteq A$ such that $I N D_{I}(B)=I N D_{I}(A)$ [69]. It is possible for a KRS to have more than one reduct. The intersection of all reducts is called the core - the set of attributes common to all reducts, essential knowledge to a KRS [21, 99, 91, 62]. 


\subsection{Variable Precision Rough Set Model, VPRSM}

The variable precision rough set model, VPRSM $[116,114,37,18,38,101,70,109]$ was originally proposed by Wojciech Ziarko in the early 1990s. It extends upon original rough set ideas introduced by Pawlak by allowing parametric definitions of rough approximation regions [57] of a decision category $X$ of interest, in a probabilistic framework which can produce a finer approximation and more accurate decisions. The spam detection system presented in Chapter 4 of this thesis applies VPRSM to build a classifier for personalized adaptive spam filtering. Theoretical aspects of the VPRSM are discussed in the subsections to follow.

\subsubsection{Email Message Representation}

In the context of the spam detection application presented in this thesis, the objects of interest will consist of a large set of email messages, $U$. This collection of emails is analyzed to extract relevant information regarding the email domain. VPRSM is employed for data analysis, to determine correlations or patterns between observations made about the objects of interest. Observations can be articulated using the concept of a probabilistic decision table which expresses stochastic dependence between two disjoint sets of atributes, particularly $C$ and $D$ (see section 3.2.4).

Data representation for thesis application used condition attributes with Boolean domains (i.e. 0,1), to reflect the presence or absence of an email feature. To achieve this, several features were discovered from email by analyzing statistical and other available information available within the message content. The domain of certain extracted features was Boolean thus directly translating to binary-valued condition attributes for decision table construction, while others were not. However, for nonboolean domain features we must first generate a set of binary-valued condition attributes acquired via a discretization process $[31,13,47,46,45]$, used to divide a range of numeric measurements into a number of subranges by properly chosen cut points. Each select cut point implies a new more relevant feature representing the ranges of values below and above it.

The spam classification presented in this thesis, applies the idea of a rough set 
approximation to the VPRSM by expressing probabilistic relations between $C$ and $D$ attributes across many tuples in a decision table. The condition attributes are predefined email characteristics representing the category of an email message. We assume here that there is only one binary-valued decision attribute, representing the category of an email message as being spam or not (i.e. legitimate). Thus there are only two decision categories corresponding to the target set, $X$ for spam emails and its complements $\neg X$ for legitimate emails.

\subsubsection{Probabilistic Knowledge}

Given the idea of a rough set approximation the VPRSM assumes that objects in universe $U$ can be statistically divided to form measurable collections of elementary sets, defined in terms of conditional attributes, to determine the indiscernibility relations amongst objects.

In addition, it is assumed that for all subsets of the universe under consideration, a description can be made about what is known about a decision attribute in the absence of condition attributes. This description can be expressed as a probability measure, the prior probability $P(X)$ of a target set $X$ (category spam in this case), such that $0 \leq P(X) \leq 1$, and can be estimated from data by evaluating the occurrence of decision category $X$ in a subset of the universe via the typical way $[6,22,110,116]$ :

$$
P(X)=\frac{|X|}{|U|}
$$

Also, assume the existence of conditional probability $P(X \mid E)$ as the probability of the occurrence of a decision category $X$, given the occurrence of an elementary set $E$ [22], estimated by:

$$
P(X \mid E)=\frac{|E \cap X|}{|E|}
$$

Next, it is assumed that for each elementary set $E$ of a determined indiscernibility relation, the probabilistic measure $P(E)$ - the support parameter for $E$, is known:

$$
P(E)=\frac{|E|}{|U|}
$$


In the equations presented above, $|*|$ denotes the cardinality of a set - the measure of the number of elements of the set. Also note that in practice, $|E|$ and $|U|$ are computed from data samples.

\subsubsection{Classification Knowledge}

With the assumptions stated in section 3.2.2, the VPRSM used for spam filter learning in this thesis application was defined in $[22,110]$. That is, the ability to form elementary sets will expose measurable probabilistic knowledge associated with subsets of the universe, which in turn reflects the VPRSM classification knowledge used to form approximate regions of a target set $X$.

Analogous to set approximations (section 3.1.2), VPRSM divides the universe into three disjoint approximate regions, namely: positive, negative and boundary. Specific to VPRSM, conditional probabilities $P(X \mid E)$ are the decisive factors for defining approximate regions, based on two precision control parameters $[114,6,86$, $114,22,110,116]$ whose select values are restricted by the prior probability $P(X)$ of the decision category $X$.

The first parameter, the lower limit $l[116]$, such that $0 \leq l<P(X)<1$, reflects the highest measure of conditional probability $P(X \mid E)$ to classify an elementary set $E$ as a subset of the negative region of the target set $X$. The second parameter [116], the upper limit $u$, such that $0<P(X)<u \leq 1$, represents the least measure of conditional probability $P(X \mid E)$ to classify an elementary set $E$ as a subset of the positive region of the target set $X$.

Simply stated, VPRSM defines the positive region of the set $X$ (the definition of spam emails), as the union of those elementary sets formed in the collection of email messages $U$ with relatively high conditional probabilities, enough to conclude that a sufficiently high proportion of messages is spam. In contrary, the negative region of $X$, as is defined as the union of those elementary sets in $U$ with relatively low conditional probabilities, enough to conclude that a sufficiently low proportion of messages in the group is spam - therefore objects in those subsets will be considered to be likely legitimate emails.

This leaves us with those elementary sets in $U$ where the conditional probability 
measures are not sufficiently high or low enough to be ruled as falling into either the positive nor negative regions of an approximation. Such subsets of the universe are described as belonging to the boundary region of the decision category $X$. The boundary can be thought of as a region designated to messages whose conditional probabilities do not significantly differ from the prior probability of sampled spam messages $P(X)$.

Formally, the positive region, negative region and boundary region of the set $X$, denoted $P O S_{u}(X), N E G_{l}(X)$ and $B N R_{l, u}(X)$ respectively, are defined by the VPRSM as [22]:

$$
\begin{gathered}
P O S_{u}(X)=\cup\{E: P(X \mid E) \geq u\} \\
N E G_{l}(X)=\cup\{E: P(X \mid E) \leq l\} \\
B N R_{l, u}(X)=\cup\{E: l<P(X \mid E)<u\}
\end{gathered}
$$

In the context of this thesis spam application, the decision category $X$ represents the decision category - spam. As such $P O S_{u}(X)$ represents those objects approximately classified as spam due to a relatively high proportion of spam amongst them. $N E G_{l}(X)$ are those not classified as spam due to a relatively low proportion of spam amongst them, therefore representing the decision category - legitimate. $B N R_{l, u}(X)$ corresponds to those objects not classified as spam or legitimate.

\subsubsection{Hierarchical Structuring of Probabilistic Decision Ta- bles}

The VPRSM represents a decision algorithm in a tabular form as probabilistic decision tables $[106,108]$, formed based on the notion of a decision table as introduced by Pawlak[57]. Probabilistic decision tables represent probabilistic associations between condition and decision attributes.

Given a decision category $X \in U / D$ and the precision control parameters, the lower $l$ and upper $u$ limits, a probabilistic decision table, denoted as $D T_{l, u}^{C, D}$, can be 


\begin{tabular}{|c||c|c|c|c||c|c|c||c|c|c|}
\hline & $\begin{array}{c}C_{1} \\
\text { flag } \\
\text { spam } \\
\text { exploits }\end{array}$ & $\begin{array}{c}C_{2} \\
\text { flag } \\
\text { reply } \\
\text { exists }\end{array}$ & $\begin{array}{c}C_{3} \\
\text { longest } \\
\text { word } \\
\text { length }\end{array}$ & $\begin{array}{c}C_{4} \\
\text { No. } \\
\text { recip- } \\
\text { ients }\end{array}$ & $\begin{array}{c}\text { No. } \\
\text { emails }\end{array}$ & $\begin{array}{c}\text { No. } \\
\text { spam }\end{array}$ & $\begin{array}{c}\text { No. } \\
\text { legit }\end{array}$ & $P(E)$ & $\begin{array}{c}P(X \mid E) \\
X=\text { spam }\end{array}$ & $\begin{array}{c}\text { VPRSM } \\
\text { Region }\end{array}$ \\
\hline$E_{1}$ & 0 & 1 & 1 & 1 & 24 & 16 & 8 & 0.3 & 0.67 & BND \\
$E_{2}$ & 0 & 0 & 0 & 1 & 24 & 1 & 23 & 0.3 & 0.04 & NEG \\
$E_{3}$ & 1 & 0 & 1 & 0 & 32 & 29 & 3 & 0.4 & 0.9 & POS \\
$\ldots$ & $\ldots$ & $\ldots$ & $\ldots$ & $\ldots$ & $\ldots$ & $\ldots$ & $\ldots$ & $\ldots$ & $\ldots$ & $\ldots$ \\
\hline
\end{tabular}

Table 3.1: Example of a partial probabilistic decision table, given $l=0.1$ and $u=0.8$

defined as a probabilistic association of each object represented by values of condition attributes, with a VPRSM approximate region as described below [22].

$$
D T_{l, u}^{C, D}(t)=\left\{\begin{aligned}
\left(P\left(E_{t}\right), P\left(X \mid E_{t}\right), P O S\right) & \Leftrightarrow E_{t} \subseteq P O S_{u}(X) \\
\left(P\left(E_{t}\right), P\left(X \mid E_{t}\right), N E G\right) & \Leftrightarrow E_{t} \subseteq N E G_{l}(X) \\
\left(P\left(E_{t}\right), P\left(X \mid E_{t}\right), B N D\right) & \Leftrightarrow E_{t} \subseteq B N D_{l, u}(X)
\end{aligned}\right.
$$

Given the equation above, $t$ is a tuple (a vector) containing values of condition attributes i.e. $t=C(e), e \in U$, and $E_{t}$ is its corresponding elementary set. In practice, defining a probabilistic decision table involves clustering objects into tuples $(\mathrm{t})$ corresponding to elementary sets, followed by computing the conditional probability $P\left(X \mid E_{t}\right)$ measures for every $E_{t}$, prior to assigning VPRSM approximation regions. Table 3.1 shows a partial probabilistic decision table, given the decision category $X=$ spam $\in U / D$, precision control parameters $(l, u)=(0.1,0.8)$, and condition attributes $C_{1}, C_{2}, C_{3}, C_{4}$.

As discussed in [22], the VPRSM uses the $\gamma(D \mid C)$-dependency measure to represent the distinctive classification of the universe in relation to decision categories $X$ and $\neg X$. This dependency measure describes the relative size of decidable subsets (i.e. $P O S_{u}(X) \cup N E G_{l}(X)$ ) and non-decidable (i.e. $\left.B N D_{l, u}(X)\right)$ subsets of the universe as presented by elementary sets formed in a decision table :

$$
0 \leq \gamma_{l, u}(X \mid C)=P\left(P O S_{u}(X) \cup N E G_{l}(X)\right) \leq 1
$$


Probabilistic decision tables are used to train and test the email domain logic for thesis spam detection application. Specifically, a linear hierarchy of decision tables can be constructed to present relationships among different combinations of candidate condition attributes relating to observed properties of sampled email messages. Hierarchies of probabilistic decision tables $[105,103]$ provide an efficient way for describing situations where varying condition attributes reveal more classification knowledge. The hierarchy structure is a more efficient approach to classification problems with high dimension space, as it increases the capability to detect more detailed facts about data with a model that is appropriate to process complex domains. The structure allows us to process data with many discovered conditional attributes, by systematically selecting a group of conditional attributes, to be combined and analyzed at each level. This hierarchical technique allows for the easy addition and removal of attributes depending on the classification task, and also increases prediction coverage by acquiring new patterns or generalizations of relationships identified, from iteration to iteration [22].

To model a hierarchy structure, the initial table is built first given the original set of condition attributes $C$ and the entire universe $U$, while every other subsequent table is derived from its parent table by defining a new universe $U^{\prime}$ as the boundary area of its parent table, and redefining the availability of the set of condition attributes $C$, by removing those attributes used in the parent level. The linear hierarchy of probabilistic decision tables will continue to grow until - either the original set of condition attributes is exhausted; no boundary area exists in a newly constructed decision table; or all tuples of a newly constructed decision table produce a boundary region-only decision table.

On each level, the $\lambda(X \mid C)$-dependency function is used to guide in the selection of $n$ condition attributes from the available pool of candidate attributes $C$, to be used to describe a defined universe $U^{\prime} \subseteq U$. Here $n$ the number of condition attributes to be selected at each level of the hierarchy is set as a system variable by administrators, similarly to the precision control parameters: $u$ and $l$. This dependency also called the normalized expected gain $0 \leq \lambda(X \mid C) \leq 1[110,107]$, is a normalized measure of the probabilistic dependency between the select subset of attributes $C^{\prime} \subseteq C$, and 
the target classification $(X, \neg X)$. In practice, the $\lambda(X \mid C)$-dependency function rules out the assumption of independence of attributes to avoid failures with problems where the degree of attribute dependencies is critical, by representing the degree of interactions between a subset of condition attributes and a target set $X$. It is defined as:

$$
\lambda(X \mid C)=\frac{\sum_{E \in U / C} P(E)|P(X \mid E)-P(X)|}{2 P(X)(1-P(X))},
$$

In theory, the point is to select a set $C^{\prime}$ consisting of the best $\mathrm{n}$ condition attributes that will produce a probabilistic decision table with elementary sets having associated conditional probabilities that most significantly differ from the prior probability $P(X)$ of $U^{\prime}$. The first member of $C^{\prime}$ is selected as the attribute returning the highest $\lambda\left(X \mid c_{i}\right)$-dependency measure computed for every $\left(U^{\prime}, c_{i} \in C, D\right)$ decision table (for $i=1, \ldots,|C|)$, then $c_{i}$ is removed from $C$. Any subsequent condition attribute of $C^{\prime}$ is selected as the attribute returning the highest $\lambda\left(X \mid c_{i}\right)$-dependency measure computed for every $\left(U^{\prime}, c_{i} \in C \cup C^{\prime}, D\right)$ decision table (for $\left.i=1, \ldots,|C|\right)$, then $c_{i}$ is removed from $C$ - reiterate (n-1) times. Note that the complexity of attribute selection is $\left(U^{2}\right)$.

The hierarchy of probabilistic decision tables can be used to generate rules from each level to be used to classify new email messages as spam or legitimate - with respect to this thesis spam predictive model. Figure 3.2 is a flow chart illustrating the hierarchical structuring of probabilistic decision tables. 


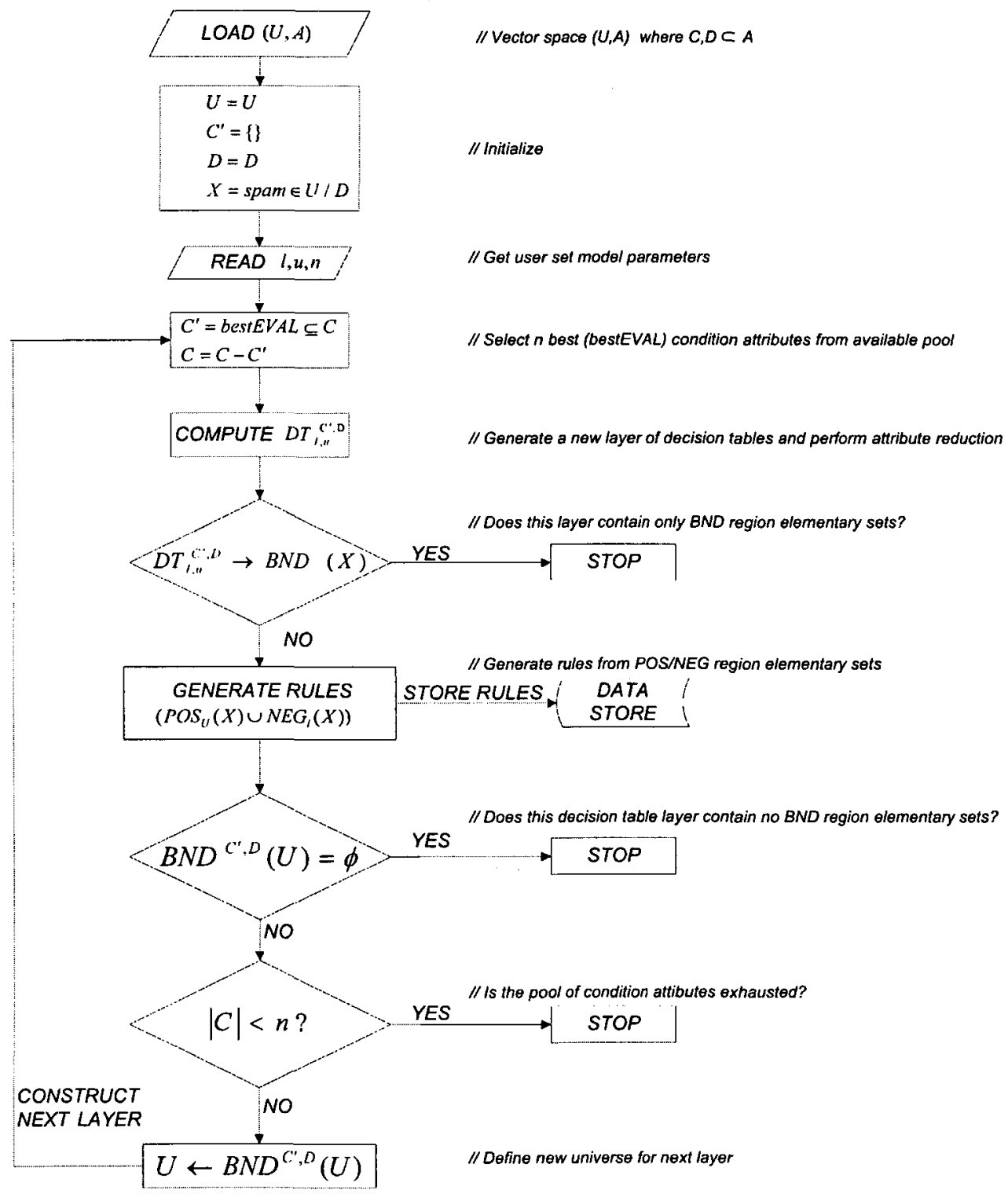

Figure 3.2: Hierarchical structuring of probabilistic decision tables 


\subsubsection{Probabilistic Decision Rules for Spam Filtering}

The spam filter system proposed in this thesis is based on learned spam detection rules computed from a structured hierarchy of probabilistic decision tables. Rules are generated at each level of hierarchy to form a pool of candidate rules, from which select rules are used to predict the category of new emails. Each rule provides a set of learned preconditions and a decision to predict the class (spam/legitimate) of message objects corresponding to the preconditions. Collectively, all learned probabilistic rules make up the classification knowledge to be used by an associated boolean classifier. Rule-based approaches are advantageous over other techniques, for example they are easily comprehensible and translated [88, 4, 63].

Probabilistic decision rules $[115,110,117,111,115,28]$ are excellent for conveying the relationship between condition and decision attributes, by translating a tabular knowledge system into a logical form. They can be described as logical implications that return a value of the decision attribute. That is a rule expresses information via values from the domains of condition attributes characterizing say an email message, and will return an outcome - if a random email message has similar characteristics, thus matching the rule.

In short, a probabilistic rule [110] is a function : $\left(C_{1}=V^{C_{1}} \wedge \ldots \wedge C_{n}=V^{C_{n}}\right) \rightarrow$ $\operatorname{category}(X)$, where $\left(C_{1} \wedge \ldots \wedge C_{n}\right)$ is a logical conjunction of attribute value pairs, and every $C_{i}$ (for $i=1, \ldots, n$ ) is an element of $\mathrm{C}$ with an associated domain value. The outcome, category $(X)$ refers to a decision category $X \in U / D$ given by the classification task at hand.

Within the context of the variable precision rough set model, every elementary set translates into a probabilistic rule [22] associated with some attached probability. Rules have the quality level in the form of the certainty gain level requirement imposed through settings of model parameters.

\section{Example 4: Rule extraction}

Given table 3.1 as an example probabilistic decision table with decision categories 
$X=$ spam and $\neg X=$ legitimate, we can extract the following probabilistic decision rules respectively corresponding to elementary sets $E_{1}, E_{2}$ and $E_{3}$ :

$$
\begin{aligned}
& \left(C_{1}=0\right) \wedge\left(C_{2}=1\right) \wedge\left(C_{3}=1\right) \wedge\left(C_{4}=1\right) \rightarrow \text { Classify as unsure, } P\left(X \mid E_{1}\right)=0.67 \\
& \left(C_{1}=0\right) \wedge\left(C_{2}=0\right) \wedge\left(C_{3}=0\right) \wedge\left(C_{4}=1\right) \rightarrow \text { Classify as legitimate, } P\left(X \mid E_{2}\right)=0.04 \\
& \left(C_{1}=1\right) \wedge\left(C_{2}=0\right) \wedge\left(C_{3}=1\right) \wedge\left(C_{4}=0\right) \rightarrow \text { Classify as spam, } P\left(X \mid E_{3}\right)=0.9
\end{aligned}
$$

Probabilistic rules can be evaluated using probabilistic measures, such as confidence factor, coverage, certainty gain and strength.

Given a probabilistic rule: $\left(C_{1} \wedge \ldots \wedge C_{n}\right) \rightarrow$ category $(X)$, let $c=\left(C_{1} \wedge \ldots \wedge C_{n}\right)$ and $d=$ category $(X)$. The confidence factor or accuracy measures how efficient a rule is in assigning an outcomd $d$, based on support data $c$. The coverage measures how well the support data $c$ describes the outcome $d$. The certainty gain measures the degree of increase in certainty of outcome $d$ using a rule $c \rightarrow d$, as opposed to simply basing decisions on what is known about the outcome $d$ in the absence of condition attributes via the prior probability $P(d)$. The strength measures the number of objects a rule refers too - that is, the possibility that support data $\mathrm{c}$ will be observable in a sampled population.

$$
\begin{gathered}
\text { ConfidenceFactor : } P(d \mid c)=\frac{|c \cap d|}{|c|} \\
\text { Coverage }: P(c \mid d)=\frac{|d \cap c|}{|d|}
\end{gathered}
$$

CertaintyGain: $P(d \mid c)-P(d), P(d \mid c)>P(d)$

$$
\text { Strength: } P(c)
$$




\section{Chapter 4}

\section{Proposed Spam Detection System}

This chapter presents a hierarchical-based variable precision rough set (HVPRS) spam detection system. It describes the design objectives of the system in 4.1, presents a system overview of the major components of the system in section 4.2 , provides a detailed discussion of the implementation of the system in sections 4.3 and 4.4 , and section 4.5 discusses system evaluation and verification of design objectives.

\subsection{System Design Objectives}

The proposed spam detection system was initially designed based on the following design goals:

- Apply HVPRS to examine a rule-based spam classification

- Design an integrative system that can efficiently combine metrics from other technical spam solutions for vast reaches of knowledge

- Apply the structure of hierarchical decision tables to efficiently manage high dimensional attribute vectors

- Produce an extensible system that allows adaptability to new spam trends

- Ensure high levels of accuracy while achieving desirable rates of unclassified cases 


\subsection{System Overview}

The operation of the spam detection system is divided into two phases: training and classification. In the training phase, a hierarchical-based variable precision rough set (HVPRS) machine learning algorithm is used on a collection of pre-classified email objects, for data generalization and rule formation. In the classification phase, classification rules acquired from training are used to predict the decision category of incoming emails. Data vectors are needed in both phases, to model (train) and test (classify) the spam detection system.

The major components of the spam detection system are described below and illustrated in figure 4.1 to follow:

- Training and Testing Datasets. This stage involves the process of retrieving and categorically labeling a large collection of hypertext email source codes (spam or legitimate). The collection of messages (corpora) is randomly partitioned into two distinct data sets; one for training the model, and the other for verifying its efficiency.

- Data Preprocessing. Given a sample of messages in data sets from the previous module, the goal here is to automatically extract structured information in some defined domains, to formally represent email messages.

- Attribute Analysis. Analyze extracted information by posing queries about some defined concepts Queries are used to formulate condition attributes for constructing a hierarchy of probabilistic decision tables. For example the concept "shouting", may be defined as the relative occurrence of emphasized text. So here analysis will be made on say html tags and characters, to investigate the usage of emphasis driven attributes say large font size, red font color, upper case letters, exclamations, etc.

- Reducing Attribute Domains. This step involves the process of transferring continuous condition attribute values into discrete counterparts via a discretisation process $[31,13]$. Discrete values are more concise to represent, easier to understand and work with, and can lead to improved predictive accuracy. 
- HVPRS Engine. This module applies the HVPRS machine learning algorithm to automatically learn generalized descriptions of elementary sets as formed in the approximation spaces of constructed hierarchies of probabilistic decision tables. Generalized descriptions are used to induce probabilistic rules for predicting the category of new incoming email.

- Classifier. Given the rules formulated in the previous module, a boolean classifier is deployed to predict the category: spam or legitimate, of new email messages.

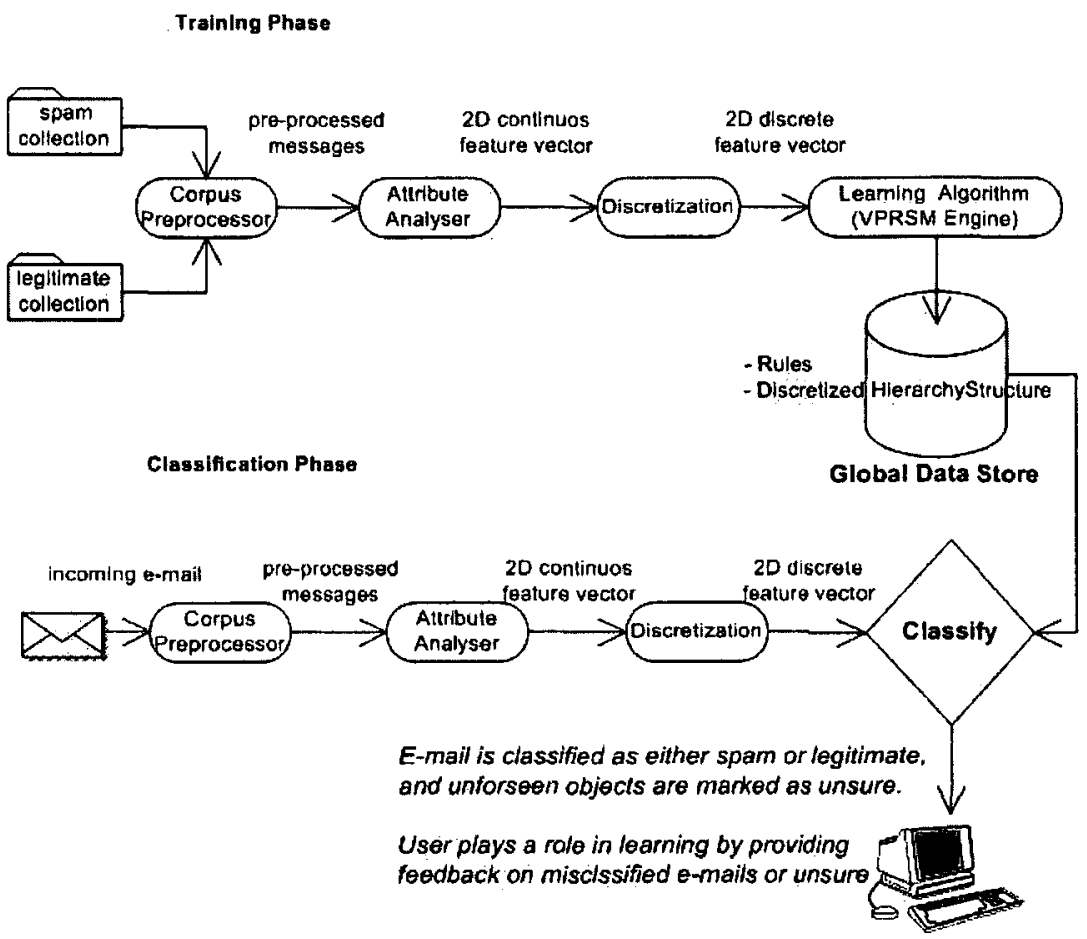

Figure 4.1: HVPRS system overview 


\subsection{Data Acquisition and Processing}

This phase of development involves the process of retrieving, understanding and labeling of hypertext documents intended for training and testing the model.

Next, information extraction techniques are applied on hypertext documents to automatically extract structured information in some domains. Extracted information is further analyzed and consolidated into attributes, which are more meaningful to the machine learning algorithm used. Tools used to facilitate this phase were Perl and MySQL.

\subsubsection{Training and Testing Datasets}

The process of obtaining all necessary data sets involves the manual retrieval and categorical labeling of a substantial number of hypertext email sources, as a complete representation of actual emails. Hypertext documents used in this application were generated from source codes obtained from a large collection of both spam and legitimate email messages.

The entire collection of email messages is randomly partitioned into two distinct groups - one for training the model, and the other for verifying its efficiency. Each individual group is then analyzed to avoid duplicate samples. Within each group, objects were divided into data sets or corpus, processed to form attribute vectors. Every training corpus formed was guided to ensure that they included a substantial amount of spam, large enough to explore many features of spam. A training corpus consisted of at least 3000 messages for a better generalization of spam, and a test corpus was limited to a minimum of 900 messages.

Since this system is intended for personalized spam filtering, legitimate emails were gathered from a single highly active user account - to avoid misclassifying a legitimate email as spam. One individual's legitimate email may be considered spam by another individual. However, spam emails were collected from multiple users' inboxes, considering that spam is sent out in bulk to multiple users so many people will receive the same spam email. Concluding that an email is spam when it is in fact legitimate is more costly than than vice-versa. 


\subsubsection{Information Extraction for Message Representation}

Prior to HVPRS-based modeling an extraction protocol was formalized for governing the standard representation of emails according to predefined representational views. These views correspond to the fields available within an email message namely: from, to, reply-to, date, cc, bcc, subject, attachment and body.

The protocol also involves extracting tokens from within an objects hypertext source for feature analysis. Individual tokens were stored in a database for further analysis. Common tokens included punctuation symbols, numbers, letters, sequences of characters, spaces, tabs, carriage returns, line feeds, and entities such as email addresses, images, symbols, html tags, attachments and URLs.

Features (inherent properties of email objects) were collected from individual and token groups within these fields, and queried to form human perceived properties of emails, used to derive attributes of A. They include: font, style, size, color, effects, spacing, length, frequencies, casing (upper, lower, mixed or proper), character (alpha, numeric or alphanumeric) and alphabetical (vowels/consonants) composition, as well as actual details surrounding html tag usage. A feature is like posing a question, so here the goal was to find answers to object-attribute related questions about email objects.

\subsubsection{Attribute Analysis}

As an extension to information extraction, analyses were performed on identified features to gather more useful information, obtained by posing queries about some defined concepts to form condition attributes. Queries were implemented as functions to probe features for indicators of spam and/or legitimate, as well as exploit attempts by spammers $[32,24]$. All computed condition attributes are pooled and used to construct the hierarchy of probabilistic decision tables described in section 4.4.1.

Considering that there may be an infinite number of ways of virtually viewing an email message, it is understandable that there are some real differences since each message is dependent on the elements that went into creating it. The first task performed at this stage is identifying possible features to be used to develop queries 
that provide a summary or descriptive view of some defined concepts relating to spam and/or legitimate messages. Interesting features were analyzed to determine structure, meaning, positioning, as well as the fraction of certain elements relative to defined categorizations in a formal grammar.

Integrity analysis involving statistical and/or logical techniques was employed to examine the blueprint of each email message, to formulate significantly logical humanperceived definitions of spam and legitimate messages, given infinite rendering variations. Heuristics were also employed to analyze exploits by spammers and indicators of both spam and legitimate emails.

To experiment the thesis spam detection application, features/attributes were formulated using relatively simple queries on extracted information. Some examples of queries used are described below:

[HtmlFontColor]

Description:- (body) Count distinct occurrences of irregular HTML font colors used in the body of an email.

Test:- Return the count of distinct color translations of any rgb, name, or hexadecimal color attribute value for all HTML $<$ font $>$ tags.

\section{[HtmlBackgroundColor]}

Description:- (body) Flag the presence of any irregular HTML background color. Test:- Analyze HTML bgcolor attribute, and other syntax (e.g. CSS) that specifies the background color of the page, for values that are not in a defined list of "regular" email background colors (e.g white). Script should consider rgb, name, and hexadecimal color values. Return true if a non-defined background color is found, else return false.

[HtmlTextChaff]

Description:- (body) Flag the presence of an HTML font color that is the same or very similar to background color. 
Test:- Get HTML font and background colors, then compare if they are the same or very similar. Compile a table indicating similar color values ( $\mathrm{rgb}$, name, and hexadecimal) to aid in comparing similarities. Return true if similar background/font colors are found, else return false.

[CountFROM]

Description:- (header) Count the number of recipients.

Test:- Return the count of comma separated values in FROM field.

$[$ CountCC]

Description:- (header) Count the number of recipients that were copied the message. Test:- Return the count of comma separated values in CC field.

\section{[Count BCC]}

Description:- (header) Count the number of recipients that were blind-copied the message.

Test:- Return the count of comma separated values in $\mathrm{BCC}$ field.

\section{[RecipientsSorted]}

Description:- (header) Flag if addresses in recipient are sorted.

Test:- Get recipient lists from To/CC/BCC fields. For each list, sort both ascending and descending and compare sorted to original list. If a sorted list is in the same order as original - return true, else return false.

[UrlCount]

Description:- (body) Count the number of links present in the body of an email.

Test:- Return the count of "href" attributes for HTML $<a>$ anchor tags.

[UsernameInOtherFields]

Description:- (header and body) Does the primary recipients' username appear in other header fields or in the body? 
Test:- Return true if a pattern match of the username is found in subject, attachment or body, else return false.

[CountImages]

Description:- (body) Count the number of images present in the body of an email.

Test:- Return the count of the number of images (pattern match for image extension e.g. jpg, png, bmp, gif).

[CountImageLinks]

Description:- (body) Count the number of image links present in the body of an email.

Test:- Return the count of the number of images (using image extension e.g. jpg, png, bmp, gif) wrapped in $<a>$ HTML tags.

$[U r l I P]$

Description:- (body) Flag an IP address in URL.

Test:- Return true if a HREF attribute has an IP address wrapped in $\langle a\rangle$ HTML tags, else return false.

[UrlUsername]

Description:- (body) Flag a username in URL.

Test:- Return true if a HREF attribute has a "username@host" pattern wrapped in $<a>$ HTML tags, else return false.

[UrlUsernamePassword]

Description:- (body) Flag a username/password in URL.

Test:- Return true if a "href" attribute has a "username:password@host" pattern wrapped in $\langle a\rangle$ HTML tags, else return false.

[UrlCharacter Encoding]

Description:- (body) Count URLs obscured with character entities. 
Test:- Return the count of the number of matched HTML character entities (consist of an ampersand, a number sign, a number and a semicolon with no spaces in between any of them) wrapped in $\langle a>$ HTML tags, else return false.

[UrlHexadecimal]

Description:- (body) Count URLs obscured with hexadecimal code.

Test:- Return the count of the number of matched hexadecimal codes (2-digit hexadecimal sequence preceded by a percent sign) wrapped in $<a>$ HTML tags, else return false.

[MessageLength]

Description:- (body) Length of the message body.

Test:- Return the arbitrary (but finite) length of the body to imply the number of characters present in the message of an email.

[NumOfWords]

Description:- (body) Count of words in the body.

Test:- Return the counts of the number of words in body. Words are defined as non null space " " separated values of a message.

[NumOfSentences]

Description:- (body) Count of sentences in the body.

Test:- Return the counts of the number of sentences in body. Sentences are defined as non null period "." separated values of a message.

[LongestWordsLength]

Description:- (body) Length of the longest word.

Test:- For every identified word in the body count the number of characters, then return the length of the longest word.

[TinyFont $]$ 
Description:- (body) Contains text with very small font size.

Test:- Return true if a pattern matches that for very small HTML font sizes in body, else return false.

[LargeFont]

Description:- (body) Contains text with very large font size.

Test:- Return true if a pattern matches that for very large HTML font sizes in body, else return false.

[Blacklisted $]$

Description:- (header) Is the senders email address blacklisted?

Test:- Return true if the sender's address is in a defined list of bad addresses, else return false.

[Whitelisted]

Description:- (header) Is the senders email address whitelisted?

Test:- Return true if an the sender's address is in a defined list of good addresses, else return false.

[CountAttachments]

Description:- (header) Number of attached files.

Test:- Return the count of the number of attachments.

[AttachNoExtension]

Description:- (header) Are there attachments with no file extension?

Test:- Return true if an attachment does not have an extension (example "filename" instead of "file.ext"), else return false.

[SizeAttachments]

Description:- (header) Size of attached files.

Test:- Return the total size of all attachments in bytes. 
[NumFileTypesAttach]

Description:- (header) Number of distinct file types.

Test:- Return the count of distinct file types (e.g. .jpg, .txt, .doc) for all attachments.

[WordStartEnd]

Description:- (header, body) Number of words that do not start or end with an alpha character.

Test:- Return the count of the number of words found that do not start or end with a an alpha character $[\mathrm{a}-\mathrm{z}]$.

[EmailFormat]

Description:- (header) Do email addresses conform to that only permissible when creating an email account?

Test:- Function to check for any malformed email addresses (e.g. if address contains no "@" or "."), also check to verify that a username contains only legal characters accepted when creating an email account.

$[$ Report Null $]$

Description:- (header, body) Report Null fields.

Test:- Report any null header or body fields.

[From $M e]$

Description:- (header) Is sender the same as recipient?

Test:- Compare from and to fields to verify whether sender is a recipient as well.

[IllegalSymbols]

Description:- (header) Does subject or the senders real name contain defined illegal symbols?

Test:- Check to see if associated fields contains any illegal symbols from a defined list. 


\section{[DigitsRatio]}

Description:- (body) the fraction of digit characters [0-9] in the message body.

Test:- If MessageLength $\neq 0$, then DigitsRatio $=$ (No. of digits $\div$ MessageLength), else DigitsRatio $=0$.

\section{[SymbolsRatio]}

Description:- (body) the fraction of symbols in the message body.

Test:- If MessageLength $\neq 0$, then SymbolsRatio $=$ (No. of symbols $\div$ MessageLength), else SymbolsRatio $=0$.

\section{[SpacesRatio]}

Description:- (body) the fraction of spaces in the message body.

Test:- If MessageLength $\neq 0$, then SpacesRatio = (No. of spaces $\div$ MessageLength), else $S$ pacesRatio $=0$.

\section{[LettersRatio]}

Description:- (body) the fraction of letters $[\mathrm{A}-\mathrm{Z}, \mathrm{a}-\mathrm{z}]$ in the message body.

Test:- If MessageLength $\neq 0$, then LettersRatio $=$ (No. of letters $\div$ MessageLength), else LettersRatio $=0$.

\section{[ConsonantRatio]}

Description:- (body) the fraction of consonants w.r.t letters in the message body.

Test:- If LettersRatio $\neq 0$, then ConsonantRatio $=$ (No. of consonants $\div$ No of letters), else ConsonantRatio $=0$.

\section{[VowelRatio]}

Description:- (body) the fraction of vowels w.r.t letters in the message body.

Test:- If LettersRatio $\neq 0$, then VowelRatio $=$ (No. of consonants $\div$ No of letters), else VowelRatio $=0$.

[AlphaNumericSymbolicRatio] 
Description:- (body) the fraction of words that contain alpha, numeric and symbolic characters.

Test:- Analyze the fraction of words containing alpha, numeric and symbolic characters w.r.t the the word count in body.

\section{[AlphaSymbolicRatio]}

Description:- (body) the fraction of words that contain alpha and symbolic characters.

Test:- Analyze the fraction of words containing alpha and symbolic characters w.r.t the word count in body.

[NumericSymbolicRatio]

Description:- (body) the fraction of words that contain numeric and symbolic characters.

Test:- Analyze the fraction of words containing numeric and symbolic characters w.r.t the word count in body.

\section{[AlphaNumericRatio]}

Description:- (body) the fraction of words that contain alpha and numeric characters. Test:- Analyze the fraction of words containing alpha and numeric characters w.r.t the word count in body.

\section{[SymbolicRatio]}

Description:- (body) the fraction of words that contain symbolic only characters.

Test:- Analyze the fraction of words containing symbolic only characters w.r.t the word count in body.

\section{[AlphaRatio]}

Description:- (body) the fraction of words that contain alpha only characters.

Test:- Analyze the fraction of words containing alpha only characters w.r.t the word count in body. 
[NumericRatio]

Description:- (body) the fraction of words that contain numeric only characters.

Test:- Analyze the fraction of words containing numeric only characters w.r.t the word count in body.

[MixedWordsRatio]

Description:- (body) the fraction of mixed cased words in the message body.

Test:- Analyze the fraction of mixed cased words w.r.t the word count in body.

[ProperWordsRatio]

Description:- (body) the fraction of proper cased words in the message body.

Test:- Analyze the fraction of proper cased words w.r.t the word count in body.

[LowerWordsRatio]

Description:- (body) the fraction of lower cased words in the message body.

Test:- Analyze the fraction of upper cased words w.r.t the word count in body.

[UpperWordsRatio]

Description:- (body) the fraction of upper cased words in the message body.

Test:- Analyze the fraction of lower cased words w.r.t the word count in body.

Feature aggregation was applied on some features to form condition attributes by constructing compound features using constructive operators on multi-elementary features. This was aimed at enhancing the discriminative power of rules generated. For example, let us consider two common exploits in spam namely - URL encoding (queries will look for hexadecimal URL, hiding target URL with an @ sign, etc.) and character encoding (\&\#109;ortgage renders into Mortgage), as features. Although both elementary features are investigated separately using distinct feature queries, one can perform an $O R$ operation on the boolean output of both queries to generate a new compound feature, represented as a new condition attribute. 
For better prediction accuracy, a large number of condition attributes were identified and pooled for growing hierarchies of the decision table, as described in the next section. This in turn produces a high dimension attribute vector consisting of many condition attributes and a single boolean decision attribute, for representing our knowledge about the email domain. Attribute vector formation is vital to system efficiency, so it is necessary to derive quality attributes representing essential properties of email objects. No feature/attribute ranking was employed at this stage since the hierarchy structure using VPRSM picks the best attributes per hierarchy level.

\subsubsection{Reducing Attribute Domains}

Condition attributes generated in the previous subsection consist of qualitative binary attributes (such as the presence or absence of images in the email) and quantitative attributes (such as the number of image links found in a message).

A functional mapping was applied to convert derived quantitative condition attributes to qualitative binary attributes for decision table construction. A common kind of mapping is dividing the range of values of an attribute into a number of suitably chosen subranges via a process called discretisation $[31,13]$. Using this process, the range of each numeric attribute was divided into a predefined number of intervals according to required representation resolution level (e.g. 20 intervals) using evenly spaced cut points. Subsequently, each cut point was treated as an attribute by itself to provide a pool of binary attributes to be used to grow the hierarchy of decision tables. The classification vectors were generated using the same cut points as those used to discretise the training samples for a given model.

\subsection{HVPRS Spam Detection System}

This section explains the two phases of the HVPRS spam detection system. The first phase is called training - the process of automatically discovering general patterns by learning generalized descriptions of elementary sets as approximated in hierarchies of probabilistic decision tables. During the second phase - classification, the learned generalized descriptions or rules are used to clasify the category, spam or legitimate, 
of incoming email messages.

\section{Training}

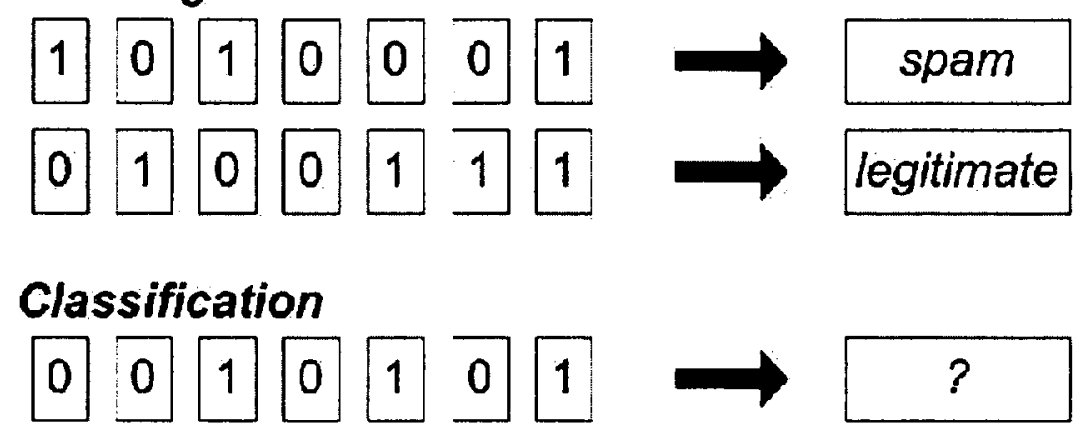

Figure 4.2: Training and classification vectors

\subsubsection{Data-Based Learning of Hierarchies of Probabilistic De- cision Tables}

A training vector consisting of thousands of email messages, is used to train a model to provide summary information regarding associated continuous and discrete randomness. Each vector is constructed following steps in the data acquisition and processing stage described in section 4.3.

VPRSM data modeling is driven, given a 2-dimensional training vector $T=(U, A)$ as a domain knowledge representation. $A$ is referred to as attributes or properties expressing acquired observations (from the previous section) about objects from the collection of emails $U$. The decision attributes objective here is to classify email objects as spam or legitimate. Predefined model control parameters are provided for each training phase, as:

- precision control constraints, lower limit $l$ and upper limit $u$, as described in section 3.2.3.

- elementary sets threshold $\phi$, used to control the minimum number of observations required to formulate a rule from a derived elementary set. 
- number of attributes per hierarchy level $n \leq|C|$, as the number of attributes to add on each hierarchy level while constructing the structure of hierarchical decision tables

VPRSM infers probabilistic knowledge represented by the probabilistic estimates associated with elementary sets formed in hierarchies of decision tables. This derived knowledge is used to construct generalized rough approximations of spam. Elementary sets represent general patterns that are automatically discovered from hierarchies of probabilistic decision tables and are used to formulate rules for predicting new incoming email.

The original vector space representing a message contains many different conditional attributes. To handle high dimensions and also increase prediction accuracy, the hierarchical model selects new attributes from the pre-computed pool of attributes at each layer of the hierarchy. The number of attributes added on each level was limited by a user-set parameter to avoid the exponential growth of the decision tables, and also to avoid the classical problem of "over-fitting" the training data. This selection process is controlled by a hill climbing [65] heuristic algorithm that attempts to maximize the degree of probabilistic dependency between the selected condition attributes and the decision attribute, using the normalized $\lambda$-dependency (equation 3.17). This selection stage is summarized in the lambda-based hill climbing (LBHC) algorithm to follow.

Given the selection of attributes for each layer, a decision table $D T_{l, u}^{C, D}$ is constructed using equation 3.15. Furthermore, for better learnable decision tables for precise rule formulation, attribute reduction was performed on each hierarchy level to eliminate any selected, but redundant conditional attributes. Reducts as introduced by Pawlak, were used to facilitate attribute reduction at this stage [57].

At every hierarchy level, rules are generated from every elementary set $\forall E_{t} \in$ $D T_{l, u}^{C, D}$, by mapping conditional attribute values to their unique VPRSM approximation region, as described in section 3.2.5. Rules generated from $\operatorname{POS}_{u}(X)$ and $N E G_{l}(X)$ elementary sets correspond to spam and legitimate decisions, respectively. However, rules obtained from $B N D_{l, u}(X)$ approximation regions were formulated based on the relation between the conditional probability of the elementary set 
$P\left(X \mid E_{t}\right)$ and the prior probability $P(X)$ such that: if $P\left(X \mid E_{t}\right)>P(X)$ then assign spam; and if $P\left(X \mid E_{t}\right) \leq P(X)$ then assign legitimate.

The major stages of the algorithm adapted for the generation of the hierarchy of decision tables are presented below. 
HVPRS $(U, C, D)$

$U$, the set of all training objects

$C$, the set of all condition attributes

$D$, the set of all decision attributes

1. $\mathbb{U} \longleftarrow U, \mathbb{C} \longleftarrow\{\}, \mathbb{D} \longleftarrow D$

2. Initialize model control parameters: $l, u, n$ and $\phi$

3. Repeat

\{

4. Select $n$ condition attributes, $\mathbb{C} \longleftarrow \boldsymbol{L B H C}(\mathbb{U}, C, D, n)$

5. Compute probabilistic decision table $D T_{\mathbb{U}}^{\mathbb{C}, \mathbb{D}}$ as a mapping $\mathbb{C} \longleftarrow\{P O S, N E G, B N D\}$

6. Perform attribute reduction on $D T_{\mathbb{U}}^{\mathbb{C}, \mathbb{D}}$

7. Generate rules $\forall E_{t} \in D T_{l, u}^{\mathbb{C}, \mathbb{D}}$, and add to this models rule set

8. Stop If $|C|<n$ or $B N D^{\mathbb{C}, \mathbb{D}}(\mathbb{U})=\emptyset$ or $\forall E_{t} \in D T_{\mathbb{U}}^{\mathbb{C}, \mathbb{D}}: E_{t} \in B N D(X)$

9. Define new universe for next layer, $\mathbb{U} \longleftarrow B N D^{\mathbb{C}, \mathbb{D}}(\mathbb{U})$

\} 


\section{$\mathbf{L B H C}(\mathbb{U}, \mathbb{C}, \mathbb{D}, n)$}

$U$, a selection of training objects

$C$, available pool of condition attributes

$D$, a set of all decision attributes

$n$, number of attributes to pick

1. Initialize: attributes $=\{\}$, count $=0$; bestEval $=0$, bestAttr $=\{\}$;

2. While count $<n$<smiles>[TlH]</smiles>

3. for all $c \in \mathbb{C}$

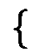

4. Set $C^{\prime} \longleftarrow c \cup$ attributes

5. If $\lambda\left(D \mid C^{\prime}\right)>$ best $E V A L$

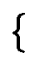

6. Set bestEval $=\lambda\left(D \mid C^{\prime}\right)$

7. Set best Attr $=c$

\}

\}

8. Set attributes $=$ attributes $\cup$ bestAttr

9. Set bestEval $=0$

10. Remove selected condition attribute from pool i.e. $C=C$ - best Att

\}

11. Return (attributes) 


\subsubsection{Rule-Based Spam Classification}

The spam detection system is comprised of a set of learned probabilistic rules and an associated boolean classifier.

The set of rules is automatically learned from hierarchies of probabilistic decision tables during the training phase, as described in the previous section 4.4 .1 , and a classifier is deployed during the classification phase to predict the category (spam or legitimate), of a vector representation of new email message(s).

Let $U=\left\{e_{1}, e_{2}, \ldots, e_{n}, \ldots e_{|e|}\right\}$ and $V_{D}=\{$ spam, legitimate $\}$, where $e_{n}$ is the $\mathrm{n} t h$ message and $D$ corresponds to decision categories. Given a message $e_{n}$, the task of the classifier is find a rule from the set of learned rules, with preconditions existing within attributes extracted from $e_{n}$. Message $e_{n}$ is classified as spam if a discovered rule assigns true, or legitimate otherwise. 


\subsection{System Evaluation}

This section discusses methods used to evaluate the effectiveness of the spam detection system, based on experimental training and classification tasks performed as described in section 4.4 .

\subsubsection{Data sets Used}

Emails collected from Microsoft Hotmail accounts were used as experimentation data sets, for both the training and classification phases. Each data set represented as a vector (U,A) consisted of a substantial number of email objects - associated with multiple condition attributes and a single decision attribute, extracted as described in section 4.3. The target set $X$ corresponds to the decision category, implicated from the boolean decision attribute's domain where $0=$ legitmate and $1=$ spam.

Individual data sets used to model (train) and test the spam detection system were derived over different time periods. Each test vector used to verify a trained model is generated using the same cut points as used to discretise its training sample. Figures 4.3 and 4.4 below illustrate time lines and approximate object counts, for all training and testing data sets used to provide system evaluations in this section.

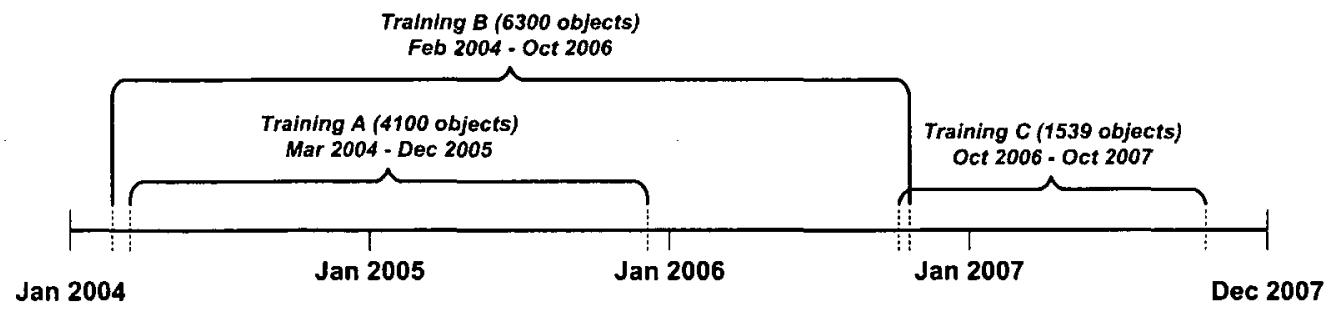

Figure 4.3: Timeline for training data sets 


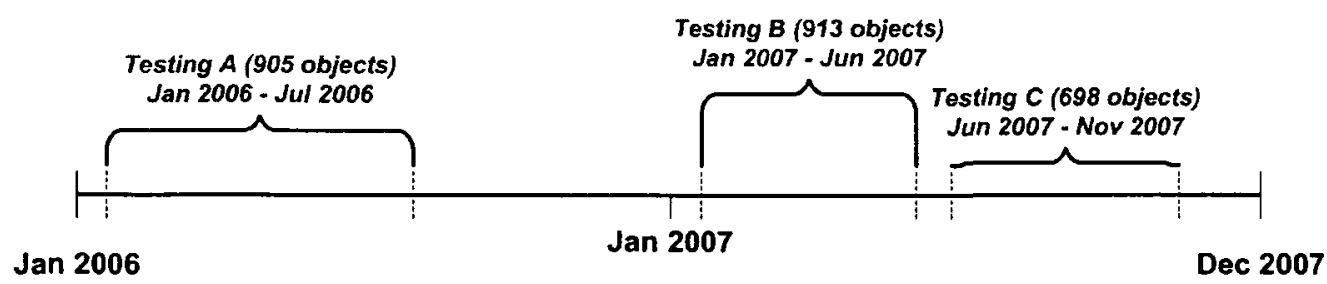

Figure 4.4: Timeline for testing data sets

\begin{tabular}{|c|c|c|}
\hline & Spam is Correct & Legitimate is Correct \\
\hline Assigned Spam & $a$ & $b$ \\
\hline Assigned Legitimate & $c$ & $d$ \\
\hline
\end{tabular}

Table 4.1: Contingency table for a binary decision category

\subsubsection{Effectiveness Measures}

Statistical measures were employed to judge results of the spam categorization experiments. Measures reported were calculated based on a $2 \times 2$ contingency table illustrated in table 4.1 above.

The values $a, b, c$, and $d$ are tallies reflecting how the assigned categories matched the correct categories for an entire testing dataset. With an objective of classifying email objects as spam or legitimate, $a$ denotes the number of objects correctly assigned as being spam (true positives); $b$ denotes the number of objects incorrectly assigned as being spam (false positives); $c$ denotes the number of objects incorrectly rejected as being spam (false negatives); and $d$ denotes the number of objects correctly rejected as being spam (true negatives).

Given the contingency table as described above, the system's effectiveness was measured using four statistical indicators: - accuracy, error, precision and recall [84]. Accuracy measures the portion of all decisions that were correct decisions; error measures the portion of all decisions that were incorrect decisions; precision measures the portion of the assigned categories that were correct; and recall measures the portion of the correct categories that were assigned.

These statistical measures can all be expressed as percentages, where $100 \%$ is the best score for the measures accuracy, precision and recall; with $0 \%$ being the best 
score for error. Note that the measures for accuracy and error are inversely related.

Based on table 4.1, measures used to evaluate the classification system are defined as follow:

$$
\begin{gathered}
\text { accuracy }=\frac{a+d}{a+b+c+d} \\
\text { error }=\frac{b+c}{a+b+c+d} \\
\text { precision }=\frac{a}{a+b} \\
\text { recall }=\frac{a}{a+c}
\end{gathered}
$$

\subsubsection{Experimental Results}

Given different predefined model control parameters for system learning (as described in section 4.4.1), table 4.2 presents sample evaluations of the proposed classification system, using training vectors A and B for modeling the classifier, and testing vector $\mathrm{A}$ for verification.

Two variations of classification measure are reported in table 4.2. The first, classification I, presents evaluations using rules learned or generated via all elementary sets, while the second, classification II, presents evaluations using rules approximated from elementary sets in positive and negative regions only. Measures reported here include: classified (the percentage of email objects whose attribute value combinations match any learned rules, generated using a trained hierarchical model), and 


\begin{tabular}{|c|c|c|c|c|c|c|c|c|c|c|c|}
\hline \multicolumn{4}{|c|}{ Trained Model } & \multicolumn{4}{|c|}{ Classification I } & \multicolumn{4}{|c|}{ Classification II } \\
\hline Vector & $1, \mathrm{u}$ & $\mathrm{n}$ & $\phi$ & Classified & Accuracy & Precision & Reciall & Classified & Accuracy & Precision & Recall \\
\hline $\mathrm{B}$ & $0.4,0.6$ & 10 & 8 & 99.12 & 97.21 & $\overline{94.90}$ & 92.54 & $98 . \overline{45}$ & 97.31 & 95.26 & $92 . \overline{35}$ \\
\hline B & $0.1,0.9$ & 8 & 5 & 100.00 & 97.13 & 94.17 & 93.27 & 96.35 & 98.39 & 100.00 & 92.51 \\
\hline B & $0.3,0.7$ & 8 & 10 & 99.67 & 97.12 & 94.09 & 93.17 & 97.13 & 97.72 & 96.67 & 92.55 \\
\hline B & $0.2,0.8$ & 5 & 10 & 100.00 & 96.80 & 95.43 & 90.38 & 97.46 & 97.62 & 99.43 & 89.64 \\
\hline A & $0.1,0.9$ & 8 & 5 & 100.00 & 95.91 & 91.71 & 90.38 & 94.03 & 97.65 & 100.00 & 88.30 \\
\hline A & $0.2,0.8$ & 8 & 5 & 100.00 & 95.80 & 96.70 & 84.62 & 96.80 & 96.35 & 100.00 & 82.70 \\
\hline A & $0.1,0.9$ & 8 & 5 & 99.78 & 95.79 & 96.67 & 84.47 & 92.04 & 97.96 & 100.00 & 89.94 \\
\hline A & $0.1,0.9$ & 8 & 10 & 98.67 & 95.52 & 96.43 & 82.65 & 92.27 & 97.72 & 100.00 & 88.89 \\
\hline A & 0,1 & 8 & 10 & 100.00 & 95.36 & 95.11 & 84.13 & 19.67 & 100.00 & 100.00 & 100.00 \\
\hline A & $0.2,0.8$ & 8 & 10 & 99.67 & 95.23 & 87.85 & 91.71 & 94.81 & 97.09 & 95.29 & 90.50 \\
\hline
\end{tabular}

Table 4.2: Sample classification experiments and reported effectiveness measures

three effectiveness measures namely - accuracy, precision and recall, as described in the previous section 4.5.2.

The average effectiveness measures over various experimental classification tasks are presented in table 4.3 below. Note that metrics reported in each row are the average measures, given a minimum of five distinct combinations of model parameter settings, for the corresponding train/test data vectors.

The first column - training, specifies the training vector (see figure 4.3) used to learn (with different combinations of model parameter settings) a set of probabilistic decision rules. The second - testing, indicates the classification vector (see figure 4.4) used to validate the probabilistic decision rules that were derived using the corresponding training model.

The measures - accuracy, precision, and recall reoccur under three groups. The first, Classification I indicates classification measures using only rules generated from positive $\left(P O S_{u}(X)\right)$ and negative $\left(N E G_{l}(X)\right)$ region elementary sets. Classification II shows categorization measures using only boundary $\left(B N D_{l, u}(X)\right)$ region rules, and the last classification III, presents measures using rules generated from all elementary sets. 


\begin{tabular}{c|c||ccc|ccc|ccc}
\hline \multicolumn{2}{c||}{ Vectors Used } & \multicolumn{3}{c|}{ Classification I } & \multicolumn{3}{c|}{ Classification II } & \multicolumn{3}{c}{ Classification III } \\
\hline Training & Testing & Accuracy & Precision & Recall & Accuracy & Precision & Recall & Accuracy & Precision & Recall \\
\hline \hline A & A & 97.32 & 99.32 & 87.74 & 71.15 & 93.35 & 82.28 & 95.33 & 93.35 & 85.94 \\
& B & 83.92 & 95.05 & 62.30 & 82.77 & 92.28 & 65.07 & 85.11 & 92.28 & 60.30 \\
\hline & A & 97.76 & 97.84 & 91.76 & 71.53 & 94.65 & 100.00 & 97.06 & 94.65 & 92.34 \\
B & B & 86.58 & 98.87 & 57.10 & 94.58 & 98.67 & 96.32 & 86.63 & 98.67 & 59.03 \\
& C & 85.03 & 100.00 & 68.27 & 99.04 & 99.90 & 100.00 & 85.24 & 99.90 & 69.30 \\
\hline \multirow{3}{*}{} & A & 95.24 & 85.24 & 89.87 & 45.93 & 62.57 & 85.28 & 85.08 & 62.57 & 87.50 \\
C & B & 97.51 & 95.97 & 95.37 & 61.71 & 83.62 & 95.95 & 92.18 & 83.62 & 95.17 \\
& C & 95.98 & 98.18 & 92.26 & 69.16 & 88.20 & 94.50 & 90.46 & 88.20 & 93.13
\end{tabular}

Table 4.3: Average effectiveness measures

\subsubsection{Analysis of Results}

Overall, the results were encouraging as effectiveness measured across different training/classifications experiments was relatively high, and therefore acceptable. Experiments demonstrated that with consistent retraining, better system efficiency is attained. For almost all tests, low rates of unclassifiable cases were observed, relative to the total number of objects classified given a testing data set. The inclusion of boundary region rules in the classifiers rule set, will generally produce greater generalization of email objects, given an associated training data set. In most cases this led to a reduction of the observed number of unclassifiable cases, thus producing a higher proportion of classified objects, as compared to using only positive and negative region rules in the classification rule set. However, the extraction of rules obtained from boundary regions had the overall impact of increased false classifications or errors, as such rules result in decision certainty just slightly better than decisions guided only by prior probabilities $P(X)$ and $\neg P(X)$.

Figure 4.5 presents a graphical view of classification measures, based on thirty-one training/classifications experiments, with varying model control parameters, sorted according to the best true classifications or accuracy. 


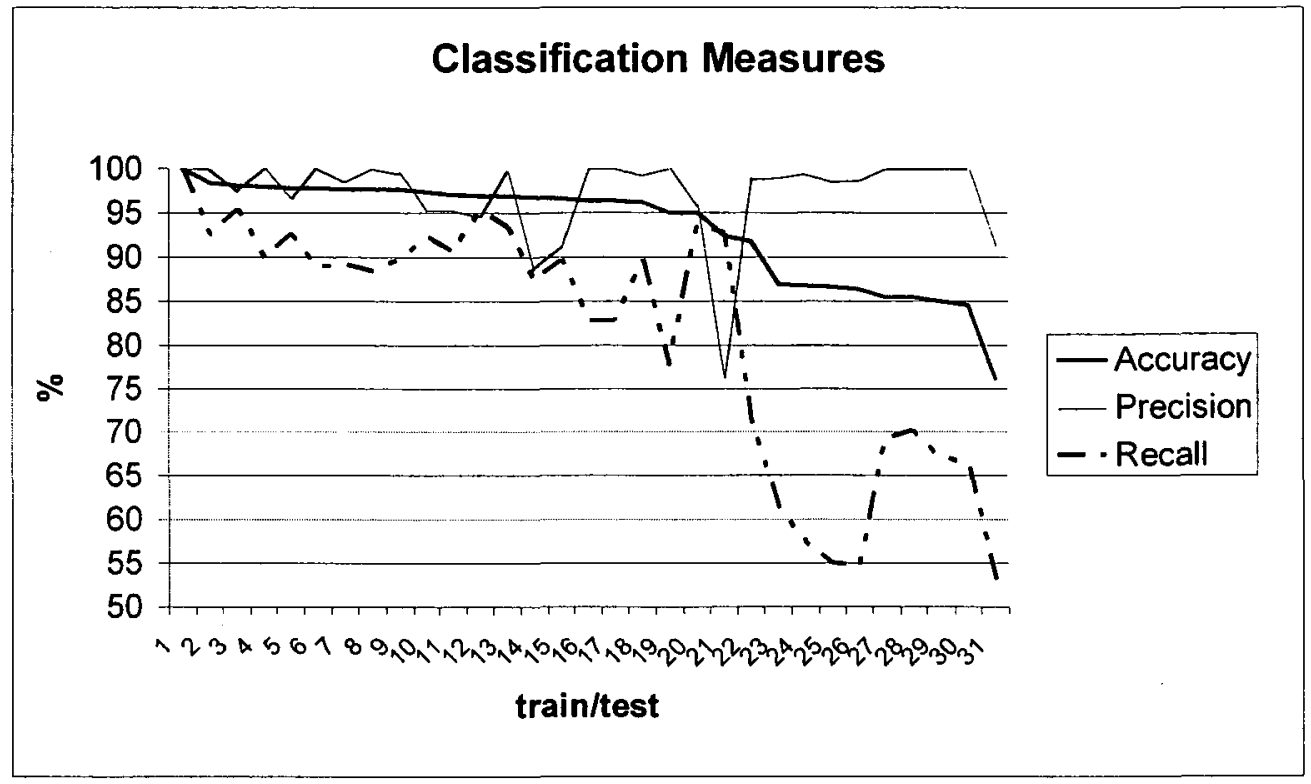

Figure 4.5: Effectiveness measures for the best accurate results

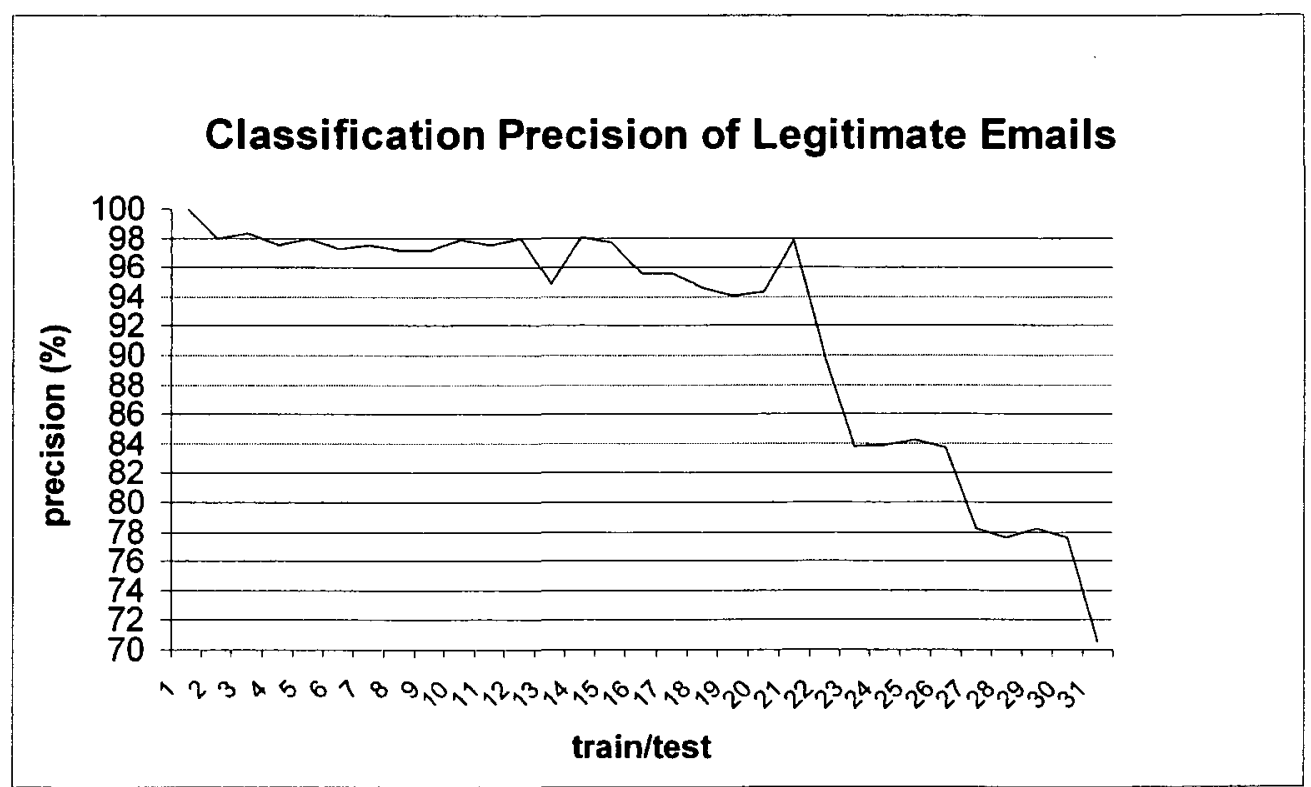

Figure 4.6: Cost analysis using the precision of legitimate emails 
Instinctively, for real time spam filtering it is preferred to classify all observed email messages as legitimate - to avoid classifying a legitimate email as spam, rather than the converse action.

Exclusive to the task of measuring the effect of misclassifying legitimate emails as spam, assume that a false positive represents a legitimate email classified as spam. To capture the cost associated with such a classification error, a measure of the precision with respect to legitimate emails was calculated to investigate the portion of the assigned categories that were correct. Sample results are illustrated in figure 4.6, for the best cost effective train/classification experiments. For a given classification task, the higher the precision - the lower the cost. These measures can be improved upon in future research relating to the optimization of system settings aimed at adjusting the decision boundary, to achieve the best results for a given model.

Various experiments were performed with or without retraining the model, and with varying model control settings. Classifiers were inspected to investigate the effects of varying specific system constraints (regardless of other parameter settings) on performance. Figures 4.7 - 4.9 below show examples of the effects of varying variables $(1, u)$, elementary set thresholds, and the number of attributes per hierarchy level, respectively, for randomly selected train/test models. For each illustration, all unmentioned model control settings remained constant, as the specified parameter will contrast to reflect the effect of their change. 


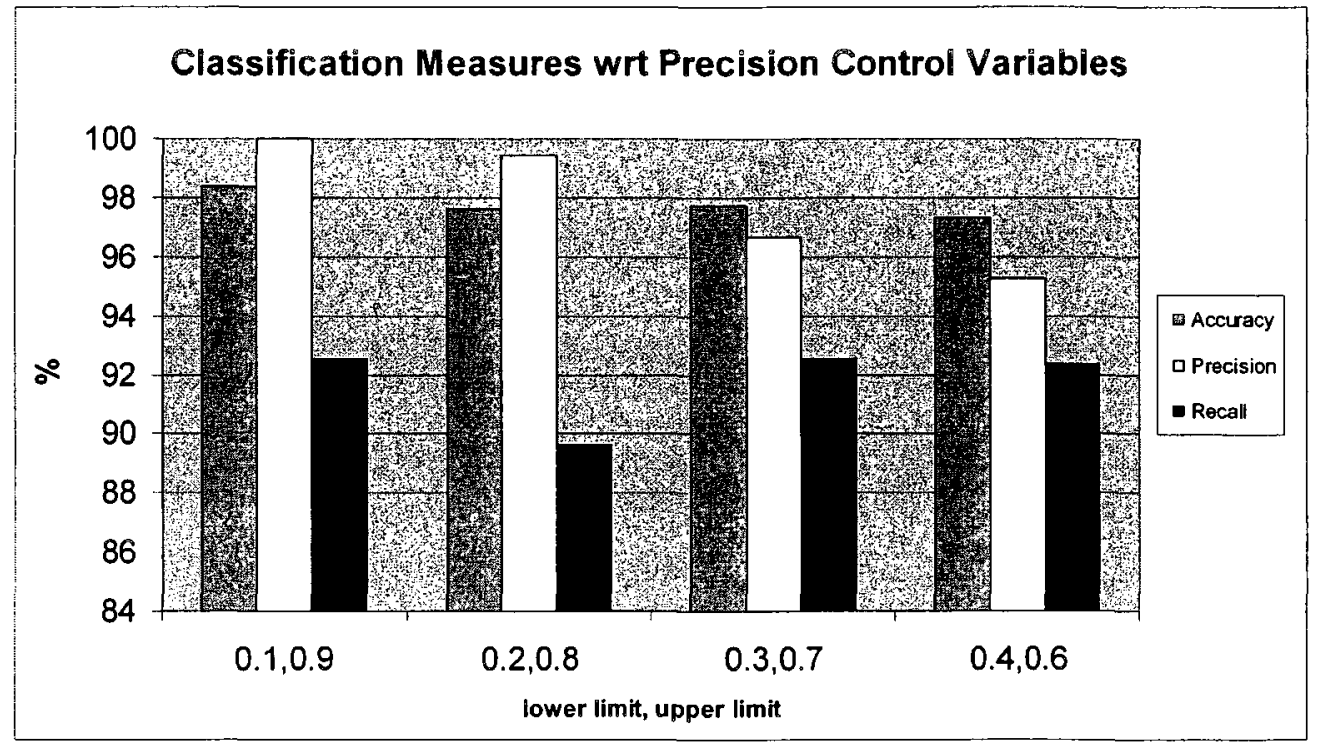

Figure 4.7: Effects of $(l, u)$ on classification measures

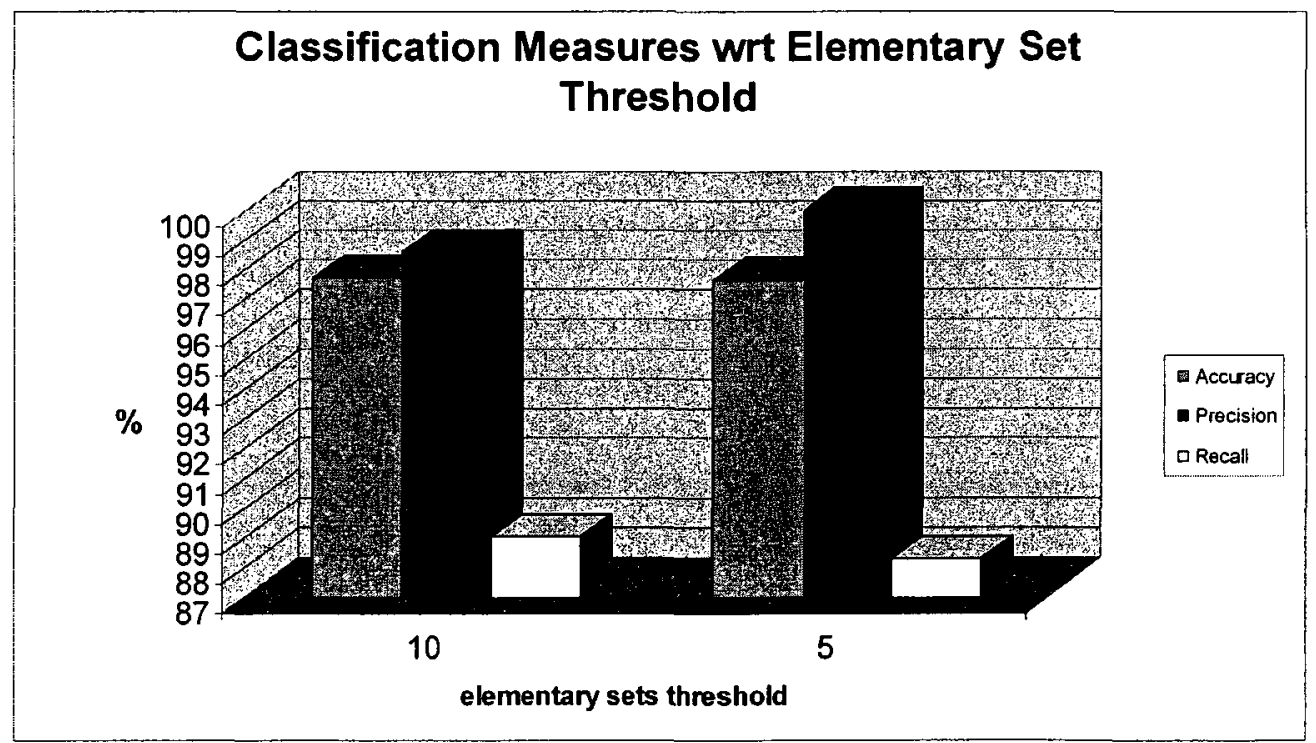

Figure 4.8: Effects of elementary set thresholds on classification measures 


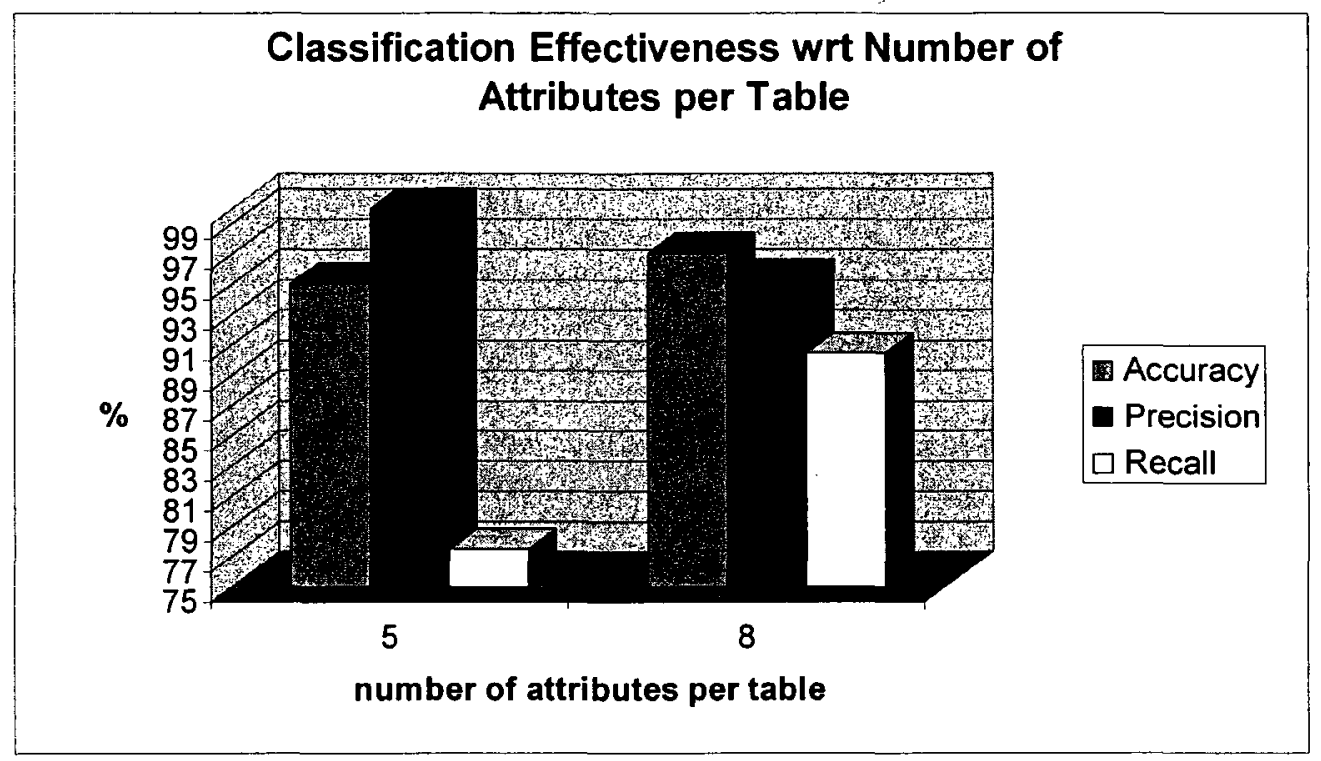

Figure 4.9: Effects of number of attributes on classification measures

A variety of test cases supply evidence that an optimal choice and combination of model control parameters is very vital, as they lead to varying classification outcomes. For example, for a given train/test classification task, when the number of attributes per table level was increased from eight to ten, this led to the increase of unclassified cases by about $10 \%$, due to the related exponential increase in the complexity of learning, or the upper bound on the maximum size of the learned decision tables. The optimization of all model control parameters to achieve the highest improvement in prediction remains a topic for further research.

\subsubsection{Confirmation of System Design Objectives}

The spam detection system was initially designed based on five main requirements outlined in section 4.1. These requirements are used to frame this section; the degree to which the system was proven in each area is discussed below.

- Apply HVPRS to examine a rule-based spam classification. It was determined that rough set/VPRSM can be efficiently applied to the spam problem, by classifying emails as spam or legitimate using rules learned from training samples. 
Using hierarchies of probabilistic decision tables allows the system to adapt to the data being applied and does not require much manual tuning to initialize and maintain. Overall the system proves to produce mostly high accurate predictions, while better performance may be attained given increased system optimization and intelligent end user collaboration.

- Design an integrative system that can efficiently combine metrics from other technical spam solutions for vast reaches of knowledge. A valid reason to work with a combination of different analysis is to provide resistance to attack. Finetuning one single technique to perfection will only encourage spammers to devise a mechanism to defeat it. With the HVPRS modeling algorithm, system administrators can incorporate spam indicators from other technical spam solutions, to be analyzed for attribute formation. For example, personal white listing and blacklisting can be integrated into condition attribute analysis to help reduce false positives.

- Apply the structure of hierarchical decision tables to efficiently manage high dimensional attribute vectors. Many problems in machine learning involve high dimensional descriptions of input features. The hierarchy structure does indeed provide a means to deal with applications where a lot of features may be needed for consideration. Instead of selecting only a few features to use for generalization, this approach allows for the collection of an initial pool of discovered features, which in turn is used to grow the hierarchy of decision tables, producing concise generalizations for shorter and more precise rule formation.

- Produce an extensible system that allows adaptability to new spam trends. Due to the complex nature of the Email domain, spammers are able to continuously mechanize exploits to beat many statistical filters, hence the need to incorporate new spam trends as they emerge. This system provides extensibility via the easy addition of new condition attributes without much manual tuning, since the hierarchy structure accommodates growing number of attributes, without doubling the number of rules. Simply, queries are developed to extract information pertaining to new spam trends, for new features/attributes formation, 
and a model is retrained with the new addition to data.

- Ensure high levels of accuracy while achieving desirable rates of unclassified cases. With this probabilistic solution, certainty thresholds are adjustable to ensure desired levels of rule accuracy, unclassifiable cases, and false positive/negative decisions. For example, system users set optimal training parameters to achieve desired rates of false positives to false negatives, without significantly increasing the number of unclassified cases. For objects falling into positive or negative regions of the decision category, the classifications produced by the properly constructed and validated hierarchy of decision tables resulted in a significantly higher rule decision certainty, or significantly lower misclassification rates as compared to decisions guided only by prior probabilities. 


\section{Chapter 5}

\section{Conclusion}

This chapter concludes my thesis. A summary of the proposed system is provided, followed by proposals for future work.

\subsection{Summary}

Experiments conducted using the hierarchical-based variable precision rough set machine learning, prove that the proposed system can be effectively applied to the email spam problem. This technique is most applicable in discovering all potentially interesting classification rules, as computed from databases containing a multiplicity of information, gathered from analyzing and querying large samples of email messages. Rules computed were short and precise, producing high prediction accuracies for several test cases. Overall, the performance of this technique was evaluated in regard to its classification accuracy across different test data sets, and most results prove relatively highly accuracies, with very low counts of unclassifiable cases. This approach generates rules with sufficient coverage and high precision, with the ability to identify the small variances in data. Also, accuracy improves overtime via retraining and the effectiveness of this technology seems hardly degradable by spammers' exploits; making it a suitable choice for a dependable spam classifier.

The hierarchy structure of this model allows for a growing number of attributes which enables systems administrators to add new features as they emerge. This advantage is particularly useful to the spam problem considering the continuously emerging exploits mechanized by spammers. New exploits or categorical indicators 
can be added to the model as they emerge, and then the model is retrained to reflect this new information.

As an integral tool in this technology, VPRSM applies probabilistic analysis to refine the choice of deterministic events, which in turn assists system administrators to fine tune the system via parameter settings for better probabilistic analysis. Various tests were performed with or without a retrained model, and with varying parameter settings. Results showed that introducing certainty parameters such as $l$ and $u$, definitely improved classification results and some combined optimization will lead to the highest improvements for a given training data set. This optimization of certainty control parameters was however not examined in this thesis.

During the information extraction stage for experimentation, very simple descriptions of the email domain were obtained; regardless, this approach still resulted in satisfactory results. For deployment, message representation should attempt to obtain better descriptions of the mail domain for more superior generalizations and rule formation. Incorporating intelligent end user learning during deployment of such a system could also increase performance.

\subsection{Future Research}

Real-time deployment of this proposed system will be considered in the next stage of research. This will apply the experimentation methodology to produce an improved version suitable for real time personalized spam filtering. Future work will first focus on developing a more robust classifier by tweaking and improving on the experimental model for an even better classification system, with increased system effectiveness and speed, coupled with optimal computational complexities, storage and operational time phases.

During the design and implementation of the system components described in this thesis, not all considerations were examined due to time constraints. However much consideration has been given to future work in the following:

1. Optimization of model control parameters (i.e certainty control parameters), elementary sets threshold and number of attributes per table, leading to the 
highest improvements for a given model.

2. Acquiring more in-depth but clear descriptions of the email domain for better generalizations and rule formations. This will include more advanced lexical analysis for natural language processing. As well, image filtering will be investigated to block image spam, where the text of a spam message is represented in an image file and displayed as a picture.

3. Integrating intelligent end user collaboration into system architecture, allowing end users to make their own judgments about email spam.

4. Incorporating better cost-sensitive evaluation when measuring performance, with an underlying assumption that classifying a legitimate email as spam, is more costly than classifying a spam email as legitimate.

5. Increasing the performance of the proposed implementation to arrive at a version with a faster implementation. 
Appendix A

\section{Table of Mathematical Symbols}




\begin{tabular}{|c|c|}
\hline SYMBOL & DESCRIPTION \\
\hline $\bar{U}$ & The universe of discourse. A set containing all objects of a problem domain under consideration \\
\hline$A$ & The set of attributes defining the properties or features of objects in $U$ \\
\hline$C$ & Condition attributes, $C \subseteq A$. Distinguished input attributes \\
\hline$D$ & Decision attributes, $D \subseteq A$. Distinguished output attributes \\
\hline$(U, A)$ & Knowledge base. Object data representation for knowledge management. Collection of objects with their attribute values \\
\hline$(U, A, C, D)$ & Specialized knowledge base with distinguished condition and decision attributes \\
\hline & Equivalence relation. A relation between objects deemed as being equivalent thus belonging to the same category \\
\hline$[x]_{R}$ & A category in $R$ containing an element $x \in U$ \\
\hline$I N D(P)$ & Indiscernibility relation over $P, P \subseteq R$ such that $P \neq \emptyset$ \\
\hline$U / C$ & Partition of the universe according to condition attributes $C$ \\
\hline$U / D$ & Partition of the universe according to decision attributes $D$ \\
\hline$X$ & A target set \\
\hline $\bar{P} X$ & The lower approximation of set $X$, containing objects that are definate members of $X$ \\
\hline$\underline{P} X$ & The upper approximation of set $X$, containing objects that are possible members of $X$ \\
\hline$\neg X$ & the complement of $\mathrm{X}$ \\
\hline$N E G_{P}(X)$ & Negative region of the set $\mathrm{X}$ \\
\hline$P O S_{P}(X)$ & Positive region of the set $\mathrm{X}$ \\
\hline$B N D_{P}(X)$ & Boundary region of the set $\mathrm{X}$ \\
\hline$\alpha(X)$ & Accuracy measure for set approximation \\
\hline$P(X)$ & Prior probability of a target set $X$ such that $0<P(X)<1$ \\
\hline$P(X \mid E)$ & Conditional probability of a decision category $X$ \\
\hline$P(E)$ & Support parameter for the set $\mathrm{E}$ \\
\hline$u, l$ & Upper and lower precision control parameters for VPRSM \\
\hline$D T_{l, u}^{C, D}$ & Probabilistic decision table \\
\hline$\gamma_{l, u}(X \mid C)$ & VPRSM Gamma dependency measure \\
\hline$\lambda(X \mid C)$ & VPRSM Lamda dependency measure \\
\hline
\end{tabular}

Table A.1: Table of Symbols Used 


\section{Appendix B}

\section{Table of Abbreviations}

\begin{tabular}{|l|l|}
\hline ABBREVIATION & FULL NAME \\
\hline ARPANET & Advanced Research Projects Agency Network \\
ASCII & American Standard Code for Information Interchange \\
BCC & Blind Carbon Copy \\
CAN-SPAM & Controlling the Assault of Non-Solicited Pornography and Marketing \\
CC & Carbon Copy \\
CSS & Cascading Style Sheets \\
DNS & Domain Name System \\
ESP & Email Service Provider \\
FTC & Federal Trade Commission \\
HTML & HyperText Markup Language \\
HVPRS & Hierarchical-Based Variable Precision Rough Set \\
IMAP & Internet Message Access Protocol \\
IP & Internet Protocol \\
IRC & Internet Relay Chat \\
ISP & Internet Service Provider \\
IT & Information Technology \\
KRS & Knowledge Representation System \\
LBHC & Lambda-Based Hill Climbing \\
MDA & Mail Delivery Agent \\
MIME & Multipurpose Internet Mail Extensions \\
MTA & Mail Transfer Agent \\
MUA & Mail User Agent \\
POP & Post Office Protocol \\
RFC & Request for Comments \\
SMTP & Simple Mail Transfer Protocol \\
TCP & Transmission Control Protocol \\
URL & Uniform Resource Locator \\
VPRMS & Variable Precision Rough Set Model \\
\hline
\end{tabular}

Table B.1: Table of Abbreviations Used 


\section{Bibliography}

[1] Albitz, P., Liu, C. DNS and BIND, 5th Edition, O'Reilly Media, 2006

[2] An, A., Shan, N., Chan, C., Cercone, N., Ziarko, W. Discovering Rules from Data for Water Demand Prediction, IEEE Expert, 72-78, 1997

[3] Alpaydin, E. Introduction to Machine Learning (Adaptive Computation and Machine Learning), MIT Press, USA, 2003

[4] Apte, C., Damerau, F., Weiss, S.M. Automated Learning of Decision Rules for Text Categorization. ACM Transactions on Information Systems, 12, 3, 233-251, 1994

[5] Architectural Comparison of Enterprise Anti-spam Solutions, A Tumblweed White Paper, Tumbleweed Communication Corp, http://www.tumbleweed.com/pdfs/2394TMWD_Enterprise_Antispam_WP_07_17_03.pdf, 2003

[6] BergerJ.O. Statistical Decision Theory and Bayesian Analysis, Second Edition, Springer-Verlag, 1985

[7] Bishop, C.M. Pattern Recongnition and Machine Learning, Springer, New York, 2006

[8] Can Laws Block Spam? An Analysis of the Effect of International SpamLegislation, Vircom Inc., http://www.spamhelp.org/articles/Can_Laws_Block_Spam.pdf, 2004 
[9] Casad, J. Sams Teach Yourself TCP/IP in 24 Hours, Fourth Edition, Sams Publishing, USA, 2008

[10] Chouchoulas, A., Shen, Q. Rough Set-Aided Keyword Reduction for Text Categorisation, Journal of Applied Artificial Intelligence, 15(9), 843-873, 2001

[11] Dash, M., Liu, H. Feature Selection for Classification. Intelligent Data Analysis, Vol. 1, No. 3, 131-156, 1997

[12] Domingos, P., Pazzani, M. On the Optimality of the Simple Bayesian Classifier under Zero-One Loss, Machine Learning, 29, Kluwer Academic Publishers, Boston, 103130, 1997

[13] Dougherty, J., Kohavi, R., Sahami, M. Supervised and Unsupervised Discretization of Continuous Features, Proceeding of the 12th International Conference Machine Learning, 194-202, 1995

[14] Drake, C., Oliver, J., Koontz, E. Anatomy of a Phishing Email. Proceedings of the First Conference on Email and Anti-Spam, 2004 http://www.ceas.cc/papers2004/114.pdf

[15] Drucker, H., Vapnik, V., Wu, D. Support Vector Machines for Spam Categorization, IEEE Transactions on Neural Networks, 10, 5, 1048-1054, 1999

[16] Duda, R.O., Hart, P.E., Stork, D.G. Pattern Classification, Wiley, New York, 2001

[17] False Claims in Spam, A Report by the FTC's Division of Marketing Practices, http://www.ftc.gov/reports/spam/ 030429spamreport.pdf, 2003

[18] Fei, X., Ziarko, W. VPRSM Approach to Web Searching, Proceedings of the Third International Conference on Rough Sets and Current Trends in Computing, Springer-Verlag, London, UK, 514-521, 2002

[19] Fontana, J. Tallying the True Cost of spam, Network World, http://www.networkworld.com/news/2003/1117spamcost.html, 2003 
[20] Friedl, J.E.F . Mastering Regular Expressions, 3rd Edition, O'Reilly Media, 2006

[21] Ganesan, G., Latha, D., Rao, C.R. Reduct Generation in Information Systems, Engineering Letters, 14, 2, 2007

[22] Glymin, M., Ziarko, W. Rough Set Approach to Spam Filter Learning, Proceedings of the International Conference on Rough Sets and Intelligent Systems Paradigm, LNCS, 4585, 350-359, 2007

[23] Goodman, J. Spam Technologies and Policies, http://www.research.microsoft.com/ joshuago/spamtech.pdf, 2003

[24] Graham, J. The Spammers' Compendium, http://www.jgc.org/tsc/

[25] Graham, P. Better Bayesian Filtering. http://www.paulgraham.com/antispam.html, 2003

[26] Graham-Cumming, J. The Spammers Compendium, MIT Spam Conference, http://www.jgc.org/tsc.html, 2003

[27] Gralla, P. PC Pest Control, O'Reilly Media, 1.3, 2005

[28] Grzymala-Busse, J. LERS - A System for Learning from Examples Based on Rough Sets, Intelligent Decision Support: Handbook of Applications and Advances of Rough Sets Theory, Kluwer, 3-18, 1991

[29] Hedley, S. A Brief History of Spam, Information \& Communications Technology Law, Routledge, 15(3), 2006

[30] Hu, F., Wang, G., Huang, H., Wu, Y. Incremental Attribute Reduction Based on Elementary Sets, Proceedings of the Tenth International Conference on Rough Sets, Fuzzy Sets, Data Mining, and Granular Computing, LNAI, 3641, Springer-Verlag, Berlin, Heidelberg, 185-193, 2005

[31] Huan, L., Farhad, H., Chew, L.T., Manoranjan, D. Discretization: An Enabling Technique, Journal Data Mining and Knowledge Discovery, 6(4), Springer, 393423, 2002 
[32] Hulten, G., Penta, A., Seshadrinathan, G., Mishra, M. Trends in Spam Products and Methods, Proceedings of the First Conference on Email and Anti-Spam, http://www.ceas.cc/papers-2004/165.pdf, 2004

[33] ITU Survey on Anti-Spam Legislation Worldwide. International Telecommunications Union, http://www.itu.int/osg/spu/spam/legislation/legislation_canada.html

[34] Jakobsson, M., Ramzan, Z. Crimeware: Understanding New Attacks and Defenses, Addison-Wesley, 2008

[35] Javvin Technologies. Network Protocols Handbook, Javvin Press, 12, 2007

[36] Jensen R., Shen, Q. Semantics-Preserving Dimensionality Reduction: Rough and Fuzzy-Rough Based Approaches. IEEE Transactions on Knowledge and Data Engineering, 16(12), 1457-1471, 2004

[37] Katzberg, J.D., Katzberg, P., Ziarko, W. Variable Precision Rough Sets Approach to Predicting Factors Affecting Delay Likelihoods, Proceedings of the Eighth International Conference on Industrial and Engineering Applications of Artificial Intelligence and Expert Systems, Melbourne, Australia, 731-736, 1995

[38] Katzberg, J.D., Ziarko, W. Variable Precision Rough Sets with Asymmetric Bounds. In Ziarko, W. (ed.), Rough Sets, Fuzzy Sets and Knowledge Discovery, Springer, London, UK, 167-177, 1994

[39] Kozierok, C.M. TCP/IP Guide, First Edition, No Starch Press, 2005

[40] Kraft, D., Martn-Bautista, M., Chen, J., Snchez, D. Rules and Fuzzy Rules in Text: Concept, Extraction and Usage, International Journal of Approximate Reasoning, 34(2-3), 145-161, 2003

[41] McDermott, J. The Issues, Impact and Reducing SPAM (Part 1), http://www.windowsecurity.com/whitepapers/Impact-Reducing-SPAM-

Part1.html, 2006

[42] Mitchell, T.M. Mahine Learning, McGraw-Hill, New York, 1997 
[43] Mullet, D., Mullet, K. Managing IMAP, 1st Edition, O’Reilly Media, 2000

[44] Mulligan, G. Removing the Spam: Email Processing and Filtering, AddisonWesley, Michigan, 1999

[45] Nguyen, H.S. Discretization of Real Value Attributes, Boolean Reasoning Approach, Ph.D. Dissertation, Warsaw University, 2007

[46] Nguyen, H.S., Skowron, A. Boolean Reasoning for Feature Extraction Problems, Proceedings of the 10th International Symposium on Methodologies for 16 Intelligent Systems, LNAI, 1325, Springer-Verlag, Berlin, 117-126, 1997

[47] Nguyen, H.S., Skowron, A. Quantization of Real Values Attributes: Rough Set and Boolean Reasoning Approaches, Proc. of the Second Joint Annual Conference on Information Sciences, 31-37, 1995

[48] Nishino, T., Nagamachi, M., Tanaka, H. Variable Precision Bayesian Rough Set Model and Its Application to Human Evaluation Data, Proceedings of the Tenth International Conference on Rough Sets, Fuzzy Sets, Data Mining, and Granular Computing, LNAI, 3641, Springer-Verlag, Berlin, Heidelberg 294-303, 2005

[49] Pawlak, Z. Flow Graphs and Decision Algorithms, Proceedings of the 9th International Conference on Rough Sets, Fuzzy Sets, Data Mining and Granular Computing, LNAI, 2639, Springer Verlag, Berlin, 1-10, 2003

[50] Pawlak, Z. Information Systems Theoretical Foundations, Information Systems, $6,205-218,1981$

[51] Pawlak, Z. On learning - A rough set approach. Lecture Notes in Computer Sciences, Springer-Verlag, 208, 197-227, 1986

[52] Pawlak, Z. Rough Set. Bulletin of Polish Academy of Science, 35, 253-258, 1987

[53] Pawlak, Z. Rough Sets and Flow Graphs, Proceedings of the Tenth International Conference on Rough Sets, Fuzzy Sets, Data Mining, and Granular Computing, LNAI, 3641, Springer-Verlag, Berlin, Heidelberg 2005 
[54] Pawlak, Z. Rough Sets, Decision Algorithms and Bayes Theorem. European Journal of Operational Research, 136, 181-189, 2002

[55] Pawlak, Z. Rough Sets, International Journal of Computer and Information Sciences, 11, 5, 341-356, 1982

[56] Pawlak, Z., Wong S.K.M., Ziarko, W. Rough Sets: Probabilistic Versus Deterministic Approach. International Journal of Man-Machine Studies, 29, 81-95, 1988

[57] Pawlak, Z. Rough Sets - Theoretical Aspects of Reasoning About Data, Kluwer Academic Publishers, Dordrecht, 1991

[58] Polkowski, L., Skowron, A. Rough Sets Knowledge Discovery 2: Applications, Case studies and Software Systems. Physica-Verlag, Heidelberg, 1998

[59] Polkowski, L., Skowron, A. Rough Sets in Knowledge Discovery 1. Methodology and Applications, Phisica-Verlag, Heidelberg, 1-576, 1998

[60] Poteet, J. Canning Spam You've Got Mail (That you Don't Want), Sams Publishing, USA, 2004

[61] Provos, N., Holz, T. Virtual Honeypots: From Botnet Tracking to Intrusion Detection, Addison-Wesley, 2007

[62] Qiu, G.F., Zhang, W.X., Wu, W.Z. Charaterization of Attributes in Generalized Approximation Representation Spaces, LNAI, 3461, pp. 84-93, 2005

[63] Quinlan, J.R. Simplifying Decision Trees. International Journal Man-Machine Studies, 27, 221-234, 1987

[64] Rauszer, C., Skowron, A. The Discernibility Matrices and Functions in Information Systems, Intelligent Decision Support, Handbook of Applications and Advances of the Rough Sets Theory, 11, Kluwer Academic, Dordrecht, 331-362, 1992

[65] Segaran, T. Programming Collective Intelligence, 1st Edition, O'Reilly Media, Inc., Ch 5.5, 2007 
[66] SPAM SCAMS - Filter Tips: 10 Scams to Screen from Your Email, OnGuardOnline, http://onguardonline.gov/docs/onguardonline_spam_scams.pdf, 2005

[67] Shan, N., Ziarko, W. An Incremental Learning Algorithm for Constructing Decision Rules, Proceedings of the International Workshop on Rough Sets and Knowledge Discovery, Banff, 335-346, 1993

[68] Shuai, J., Li, H. Using Rough Set and Worst Practice DEA in Business Failure Prediction, Proceedings of the Tenth International Conference on Rough Sets, Fuzzy Sets, Data Mining, and Granular Computing, LNAI, 3641, Springer-Verlag, Berlin, Heidelberg, 503-510, 2005

[69] Skowron, A., Swiniarski, R. Rough Sets in Pattern Recognition, in: Pal, S.K., Pal, A. (eds.), Pattern Recognition From Classical to Modern Approaches, World Scientific, River Edge, N.J, 2001

[70] Slezak, D., Ziarko, W. Variable Precision Bayesian Rough set Model, Proceedings of the Ninth International Conference on Rough Sets, Fuzzy Sets, Data Mining, and Granular Computing, LNAI, 2639, Springer Verlag, 312-315, 2003

[71] Spam Solutions White Paper, Presented by Net Sense IT Consulting, http://whitepapers.techrepublic.com/, 2004

[72] Spamhaus Statistics: The Top 10, The Spamhaus Project, http://www.spamhaus.org/statistics/countries.lasso

[73] Spammer-X, Posluns, J., Sjouwerman, S. Inside the SPAM Cartel, Syngress Publishing, USA, 2004

[74] Srinivasan, P. Intelligent Information Retrieval Using Rough Set Approximations. Information Processing and Management: an International Journal, 25(4), 347-361, 1989

[75] The CAN-SPAM Act: Requirements for Commercial Emailers, http://www.ftc.gov/bcp/conline/pubs/buspubs/canspam.htm, 2004 
[76] The Internet Engineering Task Force, http://www.ietf.org/

[77] The State of Spam, A Monthly Report Generated by Symantec Messaging and Web Security, http://www.symantec.com/avcenter/reference/Symantec_Spam_Report__January_2007.pdf, 2007

[78] Tipton, H.F., Krause, M. Information Security Management Handbook, Auerbach, Boca Ruton, Florida, 2007

[79] Tsumoto, S. Modelling Medical Diagnostic Rules Based on Rough Sets, in: Polkowski, L., Skowron A. (eds.), Rough Sets and Current Trends in Computing, LNAI, 1424, Springer Verlag, Berlin, 475-482, 1998

[80] Tynan, D. Computer Privacy Annoyances, O'Reilly Media, 3.3, 2005

[81] Understanding Phishing and Pharming, McAfee White Paper, http://www.mcafee.com/us/local_content/white_papers/wp_phishing_pharming.pdf, 2006

[82] Vakarelov, D. A Modal Characterization of Indiscernibility and Similarity Relations in Pawlak's Information Systems, Proceesings of the Tenth International Conference on Rough Sets, Fuzzy Sets, Data Mining, and Granular Computing, LNAI, 3641, Springer-Verlag, Berlin, 12-22, 2005

[83] Wang, J., Wang, J. Reduction Algorithms Based on Discernibility Matrix: The Ordered Attributes Method, Journal of Computer Science and Technology, 16, 489504,2001

[84] Williams, K. CPAN: Statistics - Contingency, http://search.cpan.org/ kwilliams/Statistics-Contingency-0.08/Contingency.pm, 2004

[85] Wong, S.K.M. Ziarko, W. A Machine Learning Approach to Information Retrieval, Proceedings of the Ninth Annual International ACM SIGIR Conference on Research and Development in Information Retrieval, Italy, 228-233, 1986 
[86] Wong, S.K.M. Ziarko, W. INFER - An Adaptive Decision Support System Based on the Probabilistic Approximate Classification, Proceedings of the Sixth International Workshop on Expert Systems and their Applications, Avignon, 713-725, 1986

[87] Wong, S.K.M., Ziarko, W. On Learning and Evaluation of Decision Rules in the Context of Rough Sets, Proceedings of the ACM SIGART Int. Symp. on Methodologies for Intelligent Systems, 308-324, 1988

[88] Wulthrich, B. Discovering Probabilistic Decision Rules, International Journal of Approximate Reasoning, 1993

[89] Wybraniec-Skardowska, U. On a Generalization of Approximation Space. Bulletin of the Polish Academy of Sciences, 37, 51-61, 1989

[90] Yao, Y. Probabilistic Approaches to Rough Sets, Expert Systems, 20(5), 287-291, 2003

[91] Yao, Y., Zhao, Y., Wang, J. On Reduct Construction Algorithms, Proceedings of the First International Conference on Rough Sets and Knowledge Technology (RSKT'06), 297-304, 2006

[92] Yao, Y.Y, Zhao, Y. Conflict Analysis Based on Discernibility and Indiscernibility, Proceedings of IEEE Symposium on Foundations of Computational Intelligence, 302-307, 2007

[93] Yao, Y.Y. A Comparative Study of Formal Concept Analysis and Rough Set Theory in Data Analysis, Proceedings of the Fourth International Conference on Rough Sets and Current Trends in Computing, LNCS, 3066, Springer, Berlin, 59-68, 2004

[94] Yao, Y.Y. On Generalizing Pawlak Approximation Operators, Proceedings of the First International Conference on Rough Sets and Current Trends in Computing, LNAI, 1424, 298-307, 1998

[95] Yao, Y.Y., Generalized rough set models, in: Polkowski, L., Skowron, A. (eds.), Rough Sets in Knowledge Discovery, Physica-Verlag, Heidelberg, 286-318, 1998 
[96] Yao, Y.Y., Probabilistic rough set approximations, International Journal of Approximation Reasoning, 49, 2, 255-271, 2008

[97] Zdziarski, J.A. Ending Spam, No Starch Press, San Francisco, 2005

[98] Zhang, L., Zhu, J., Yao, T. An Evaluation of Statistical Spam Filtering Techniques. ACM Transactions on Asian Language Information Processing, 3(4), 2004

[99] Zhao, Y., Luo, F., Wong, S.K.M., Yao, Y.Y. A General Definition of An Attribute Reduct, Proceedings of Second International Conference Rough Sets and Knowledge Technology, LNAI, 4481, 101-108, 2007

[100] Zhao, Y., Yao, Y.Y., Luo, L. Data Analysis Based on Discernibility and Indiscernibility, Information Sciences, 177, 4959-4976, 2007

[101] Ziarko, W. Set Approximation Quality Measures in the Variable Precision Rough Set Model, Soft Computing Systems: Design, Management and Applications, IOS Press, Santiago, Chile, 442-452, 2002

[102] Ziarko W., Shan, N. Data-Based Acquisition and Incremental Modification of Classification Rules, Computational Intelligence, 11(2), 357-370, 1995

[103] Ziarko, W. Acquisition of Hierarchy-Structured Probabilistic Decision Tables and Rules from Data, Proceedings of IEEE Intl. Conference on Fuzzy Systems, Honolulu, USA, 779-784, 2002

[104] Ziarko, W. Data Mining Tasks and Methods: Rule Discovery: Rough Set Approaches for Discovering Rules and Attribute Dependencies, Handbook of Data Mining and Knowledge Discovery, Oxford University Press, New York, 328-339, 2002

[105] Ziarko, W. Incremental Learning and Evaluation of Structures of Rough Decision Tables, Lecture Notes in Computer Science, 3700, 162-177, 2005

[106] Ziarko, W. On Learnability of Decision Tables. Rough Sets and Current Trends in Computing, LNCS, 3066, 394-401, 2004 
[107] Ziarko, W. Partition Dependencies in Hierarchies of Probabilistic Decision Tables, Proceedings of the First International Conference on Rough Sets and Knowledge Technology, LNCS, 4062, 42-49, 2006

[108] Ziarko, W. Probabilistic Decision Tables in the Variable Precision Model, Computational Intelligence, 17, 593-603, 2001

[109] Ziarko, W. Probabilistic Rough Sets, Rough Sets, Fuzzy Sets, Data Mining, and Granular Computing, Proceedings of the Tenth International Conference on Rough Sets, Fuzzy Sets, Data Mining, and Granular Computing, LNAI 3641, 283-293, 2005

[110] Ziarko, W. Probabilistic Rough Sets, Proceedings of the Tenth International Conference on Rough Sets, Fuzzy Sets, Data Mining, and Granular Computing, LNAI, 3641, Springer-Verlag, Berlin, Heidelberg, 283-293, 2005

[111] Ziarko, W. Rough Set Approaches for Discovery of Rules and Attribute Dependencies, Handbook of Data Mining and Knowledge Discovery, Oxford University Press, 328-339, 2002

[112] Ziarko, W. Rough Sets and Knowledge Discovery: An overview, in: Ziarko, W. (ed.), Rough Sets, Fuzzy Sets and Knowledge Discovery, Springer Verlag, 11-15, 1994

[113] Ziarko, W. Rough Sets: Trends, Challenges, and Prospects, Proceedings of Second International Conference on Rough Sets and Current Trends in Computing, LNCS, 2005, Springer-Verlag, Berlin, Heidelberg, 1-7, 2001

[114] Ziarko, W. Set Approximation Quality Measures in the Variable Precision Rough Set Model, Soft Computing Systems, Management and Applications, IOS Press, 442-452, 2001

[115] Ziarko, W. Shan, N. A Method for Computing All Maximally General Rules in Attribute-Value Systems, Computational Intelligence, 12(2), 223-234, 1996 
[116] Ziarko, W. Variable Precision Rough Set Model, Journal of Computer and System Science, 46, 39-59, 1993

[117] Ziarko, W. Xiao, X. Computing Minimal Probabilistic Rules from Probabilistic Decision Tables: Decision Matrix Approach, Proceedings of the Second International Atlantic Web Intelligence Conference (AWIC 2004), 84-94, 2004 